\title{
Flora of Kansai Basin at Lalgarh of Paschim Medinipur District in West Bengal with Special Reference to Eco-degradation in India
}

\author{
Dr. Debabrata Das \\ Ecology Laboratory, Lalgarh Government College Lalgarh, Paschim Medinipur-721 516, West Bengal, India \\ (Formerly Microbiology Laboratory, P.G. Deptt. of Botany, Darjeeling Govt. College, Darjeeling and Botany \\ Department, Jhargram Raj College, Jhargram, Paschim Medinipur, West Bengal)
}

\begin{abstract}
Flora is systematic enumeration of plant species in a given community of a given ecosystem. It reflects the quality as well as the health of the ecosystem because the floral community are the producers which support the growth and development of ecosystem. It reflects the inflow of nutrients and recycling processes through bio-geochemical processes. Indiscriminate use of different chemicals and articles for different purposes produced by different leading factories for the modern development of human societies causes threat to the environment and loosing the biodiversity which significantly destroying the environment rapidly. The main reason is increasing load of human population which damaging the environment and degrading the ecosystem by pollution directly or indirectly. The present paper reflects the 57 floral elements under 29 families and the community structure including eco-degradation process which could be a starting point of extension through community research by a group of workers in future. Hope that interested people will come and take that opportunity to record the problems for future study and assessment about the dwindling ecosystem for our existence at Lalgarh.
\end{abstract}

Keywords: Kansai Basin, Flora, Community composition and Eco-degradation, Sustenance

\section{Introduction}

Flora is a composition of common plant elements in and ecosystem which plays a significant role in the environment to develop community. Population is a general term that emphasised on a particular data to enumerate the flora through numerical study. The present study broadcasts Kansai Basin flora at Lalgarh of Paschim Medinipur District in West Bengal. In a static way the floral elements are not static but dynamic due to enforcement of power through nutrient flow and other stress. As the river is floating and the current is maximum during monsoon so the basin and bank elements change their composition time to time. Some parts of the Bank of Kansai River are eco-fragile so the continuous change of habitat and land pattern is going on which manifests a havoc change in the structure and composition of species and ultimately change the community composition in non-climax vegetation. Waste land nearer to the bank is going threat due to anthropogenic purposes and soil erosion is another factor that governs the havoc change hitherto to change the dynamics of vegetation. As a whole the changing habitats changing the composition from time to time also from season to season. It is because some plants become submerged or completely merged during complete flow of water during monsoon, so no vegetation was there in the river basin during monsoon to late monsoon. The only late monsoon and winter broadcasts typical vegetation mainly herbs along the moist bank of river Knasai (Kansawati) at Lalgarh of Binpur-I Community Development Block under Jhargram sub-division which is going to be Jhargram District very soon. The early summer and pre monsoon change the vegetation which having maximum phenological change due to stress prone condition. The plants get flowers and fruits as early as possible which could be due to a natural threat. The other part of the river sites e.g. high ridge river bank is very dry with no herbs during monsoon to late summer except a few cacti and other succulent shrubs along with some thorny plants. The interesting episode is that the centre of the river basin shows paddy (rice) cultivation during winter to early summer when the river having no serious water flow even the river is looking dead. A gentle flow of water discharges the typical propagules blowing from Chotanagpur plateau of Jharkhand state and causing the onset of sprouting vegetation very fast and in vivid form because the time is limited. As soon as the monsoon started, the propagules cut off the vegetative part and settle the plant above the high ridge of the bank where the settlement becomes safe to community development. The herbs available in the Basin of Kansai River are mostly ephemeral because they complete the life span very faster compared to the same plants available in other sites even some plants are now not physically visible due to scorching of heat due to non-availability of water. But interesting episode is that maximum species in embankment area presenting underground propagules for growth in the next year sprout though the aerial parts become dead. That vivid explanation may be made by lesser spear grass or love Grass (Chrysopogon aciculatus) in high ridge of river bank Kansai where they buried rhizome part 
for their presence in a safe custody. Drought escaper easily escaping drought stress and shows propagules before the onset of hot summer for their work as a challenge bit. Commas, pappus and dry achenes are good examples of such type found in Calotropis gigantea, Eupatorium odoratum and Clematis spp. very faster movement of double sided commas in Alstonia scholaris found in the same site that rapidly disseminate and establish new plant here and there. Dry and hard coated seeds found in Ziziphus spp. and Martynia sp. and even in Xanthium spp. which get easy settlement along the bank of river Kansai and sprout during the onset of monsoon. In the present study trees, herbs and shrubs of 57 species have been studied well under 29 families to know the pattern of ecological significance and role for community development at Kansai Basin of Lalgarh area in Paschim Medinipur District. Eco-degradation causing loss of ecosystem, loss of habitats and loss of species through huge collection of resource by local people there by. But there was no proper management so that the present study argued to generate traditional knowledge as well as suggestion to pristine the ecosystem healthy wealthy and sound which could be the slogan in near future to save the environment to flourish the revived ecosystem for our existence and for our sustenance.

\section{Study Area}

Study area falls under Binpur-I community development block of Jhargram sub-Division in Paschim Medinipur District of West Bengal state, India. It is a lower tract of Chotanagpur plateau with lateritic red soil along with alluvial parent rocky substances including high and low ridge. The tract is Lalgarh area along the Basin of Kansai River that flows towards Dherua of Paschim Medinipur District. The bank has river plateau having shrubberies, waste land, fallow land and undulated degraded land with high erosive soil profile. Significant loss of soil is due to flow of water during heavy monsoon and lower drainage system flow the heavy mass of soil through mullah and gulley. Extremely stiffly bank and moist community have been taken as habitat for the study of flora and ecosystem as a whole to record the vegetation along with environmental components. Only the winter season was taken in to account and study field was inclusively nearer to Lalgarh Government College and Model School of Lalgarh along the slopes and almost entirely the river basin with moist and dry community of plants in the said area.

\section{Materials And Methods}

Study was conducted in the riverbank and basin of Kansai at Lalgarh with a programme scheduled for study of ecosystem and flora only for winter season when most of the composition available as dead or almost dry. The season was winter starts since October to January with significant lower temperature i.e. 9-10 degree centigrade during January and $26-28^{0} \mathrm{C}$ during October. The degree of variation during the study period ranged between $9-28^{0} \mathrm{C}$ degree centigrade with varied moisture content in both the waste land available at high ridge of the river bank and low lying moist community nearer to flow of water at the bottom of the river. Moisture content ranged between 7 and $14 \%$ in bank soil and sandy soil respectively for the moist community. Regular visit have been made in the study sites along with the students of Botany Department of Lalgarh Government College, Lalgarh, Paschim Medinipur, West Bengal. Photographs were taken from field. Plant specimens were collected time to time to analyse the material at Ecology Laboratory of Botany Department, Lalgarh Government College. Soil was carried out to know the moisture content study. Temperature metre was used to study the temperature. Flora and monographs along with the literature was consulted to identify the plants. Herbarium specimens was prepared and housed in the laboratory of Lalgarh Govt. College for further study. Phenological studies were continued since July 2016 till date with the parity of plant material available in Jhitka Forest under Paschim Medinipur Forest Division. Some living plant materials were placed at earthen pot to know the vegetative stage of the plant under artificially nurtured condition. Literature 1-42 was used to support the document and also for further study.

\section{Results And Discussion}

The present study revealed 57 plant species under 56 genera and 29 families in which 9 plant species under monocotyledons and 48 species under dicotyledons. The ratio of monocot to dicot species found was 1:5.3. Here, highest frequency of species was encountered in case of families like Asteraceae (6) and Poaceae (5) followed by Lamiaceae (4). Lowest frequency i.e. lowest number encountered in families like Acanthaceae, Alangiaceae, Asclepiadaceae, Capparaceae, Combretaceae, Cucurbitaceae, Ehretiaceae, Martyniaceae, Meliaceae, Onagraceae, Rutaceae, Scrophulariaceae, Solanaceae and Ulmaceae (Table 1). Some plant species were very important because they provide economically significant yield as timber, fruits, flowers, flosses and whole plants as medicinal one. The high ridge had a less to lesser number of grasses as because during winter almost all sites become dry that cannot produce the vegetative growth except a few thorny plants like Solanum xanthocarpum (Fig. 6) and Ziziphus sp.. The only plant Tridax procumbens (Fig. 16) showed greenish patch along with a grass like Cynodon sp. which have immense importance to the grazed animals. Calotropis gigantea 
Flora of Kansai Basin at Lalgarh of Paschim Medinipur District in West Bengal with Special ..

was used by goat and buffaloes as fodder which was non-fodder plant for other animals. The small patch of rice field in and around the river bed showed minimal growth of Coldenia procumbens (Fig. 25) which was a anticancer plant under the family Ehretiaceae may be used for future study that have a controversial position i.e. under the family Ehretiaceae or Boraginaceae or Cordiacae. No flower or fruits have been recorded till the end of the January. Scattered patch of dry grass like Chrysopogan aciculatus (Golden false beard grass) have been recorded which have underground rhizome will revive soon during monsoon. Some important medicinal plants like Azadirachta indica, Aristolochia indica, Ichnocarpus frutescens and Hemidesmus indicus were frequently found in and around the study site during monsoon but not now seen. It could be a repository for future study and research. Some climbers like Tiliacora acuminate, Combretum decandrum and Capparis sp. were found as hedge along with important medicinal plant like Cocculus hirsutus in the study site. Small herbaceous plants like Dentella repens, Spermacoce hispida, Ocimum americanum, Eragrostis tenella, Cyperus kyllinga, Tragia hispida were found as common weeds which showed flowers but in normal condition none place showed the same plant under flowering condition. This is because all plants of moist community became ephemeral rather than normal. This could be a new angle for further study and research in the said area from different field.

Table 1. Plants of Kansai basin along with English, Bengali and family name(s)

\begin{tabular}{|c|c|c|c|c|}
\hline SL. No. & Scientific Name & English Name & Bengali Name & Family \\
\hline 1. & $\begin{array}{l}\text { Ipomoea carnea Jacq. ssp. fistulosa } \\
\text { (Mart. Ex Choisy) D. Austin }\end{array}$ & $\begin{array}{l}\text { Bush Morning Glory/Pink } \\
\text { Morning Glory }\end{array}$ & $\begin{array}{l}\text { Uridam/Jhoradan/ } \\
\text { Ha-Kalmi }\end{array}$ & Convolvulaceae \\
\hline 2. & Phoenix sylvestris L. & Date Palm & Khejur & Arecaceae \\
\hline 3. & Lippia geminate Kunth & Bushy Matgrass/Pitiona & Motmotia ful & Verbenaceae \\
\hline 4. & $\begin{array}{l}\text { Limonia acidissima L. } \\
\text { =Feronia elephantum } \\
=\text { F. limonia }\end{array}$ & Elephant-apple & Kothbel/Kaith & Rutaceae \\
\hline 5. & Saccharum spontaneum L. & Serio grass & Kans ghas & $\begin{array}{l}\text { Poaceae/ } \\
\text { Graminae }\end{array}$ \\
\hline 6. & $\begin{array}{l}\text { Solanum xanthocarpum Schard \& } \\
\text { Wendl. }\end{array}$ & $\begin{array}{l}\text { Yellow berried Night } \\
\text { shade }\end{array}$ & Kantikarii & Solanaceae \\
\hline 7. & $\begin{array}{l}\text { Pithecellobium dulce } \text { (Roxb.) } \\
\text { Willd. }\end{array}$ & Manila Tamarind & Jilapi/Jalebi & Mimosaceae \\
\hline 8. & $\begin{array}{l}\text { Vachelia nilotica (L.) P. J. H. } \\
\text { Hurter \& Mabb. } \\
=\text { Acacia nilotica (L.) Willd. ex. } \\
\text { Dellile } \\
=\text { A. Arabica (Lam.) Willd. }\end{array}$ & $\begin{array}{l}\text { Gum Arabica/ } \\
\text { Prickly Acacia }\end{array}$ & Babla/Babul & Mimosaceae \\
\hline 9. & Croton bonplandianum Baill. & Bonpland's Croton & Ban Tulsi & Euphorbiaceae \\
\hline 10. & Ziziphus sp. & Shrubby Boir & Pal Kul & Rhamnaceae \\
\hline 11. & Borassus flabellifer L. & $\begin{array}{l}\text { Asian Palmyra } \\
\text { Palm/Toddy Palm }\end{array}$ & Tal & $\begin{array}{l}\text { Arecaceaea/ } \\
\text { Palmae }\end{array}$ \\
\hline 12. & Oryza sativa L. & Asian Rice & Dhan & Poaceae \\
\hline 13. & Evolvulus nummularius (L.) L. & Agracejo/Rastrero & Bhnui-Akra & Convolvulaceae \\
\hline 14. & Cyperus rotundus L. & Nutgrass/Nut sedge & Mutha & Cyperaceae \\
\hline 15. & Desmodium triflorum (L.) DC. & $\begin{array}{lr}\text { Three } & \text { flower } \\
\text { beggarweed/ } & \text { Creeping } \\
\text { tick trefoil } & \\
\end{array}$ & Kudaliya & Fabaceae \\
\hline 16. & Tridax procumbens $\mathrm{L}$. & Tridax daisy/Coat buttons & Tridaksha & $\begin{array}{l}\text { Asteraceae/ } \\
\text { Compositae }\end{array}$ \\
\hline 17. & Spermacoce hispida L. & False button weed & Madanghati & Rubiaceae \\
\hline 18. & $\begin{array}{l}\text { Mecardonia procumbens (Mill.) } \\
\text { Small }\end{array}$ & Baby jump-up & Garur bramhi & Scrophulariaceae \\
\hline 19. & $\begin{array}{l}\text { Eclipta prostrate L. } \\
=\text { E. alba (L.) Hassk. }\end{array}$ & False daisy & Kesut/Kesta & Asteraceae \\
\hline 20. & Cassia tora L. & Sickle Pod/Sickle Senna & Jhunjhuni & Caesalpiniaceae \\
\hline 21. & Cynodon dactylon (L.) Pers. & Bermuda grass & Durba/Duba & Poaceae \\
\hline 22. & Kylinga brevifolia Rottb. & $\begin{array}{l}\text { Green head sedge/Short } \\
\text { leaf spike sedge }\end{array}$ & Nirbis & Cyperaceae \\
\hline 23. & Blumea oxyodonta DC. & - & Kukshima & Asteraceae \\
\hline 24. & $\begin{array}{l}\text { Eragrostis tenella (A.Rich.) } \\
\text { Hochst. ex Steud }\end{array}$ & $\begin{array}{l}\text { Love Grass/Feather love } \\
\text { grass }\end{array}$ & - & Poaceae \\
\hline 25. & Coldenia procumbens L. & - & Tripunkhi & $\begin{array}{l}\text { Ehretiaceae/ } \\
\text { Boraginaceae }\end{array}$ \\
\hline 26. & $\begin{array}{l}\text { Helichrysum luteoalbum } \\
\text { Rehb. } \\
\text { =Gnephalium luteoalbum L. }\end{array}$ & $\begin{array}{l}\text { Jersey Cudweed/ } \\
\text { Weedy Cudweed }\end{array}$ & Scora & Asteraceae \\
\hline 27. & Stereblus asper Lour. & Sand Paper tree & Seora & Moraceae \\
\hline 28. & Morinda angustifolia Roxb. & $\begin{array}{l}\text { Narrow leaved Indian } \\
\text { Mulberry }\end{array}$ & Daruharidra & Rubiaceae \\
\hline 29. & Azadirachta indica A. Juss. & Margosa tree & Neem/Nim & Meliaceae \\
\hline 30. & Clerodendrum viscosum Vent. & Clerodendrum & Ghetu/Glory tree & Verbenaceae \\
\hline
\end{tabular}


Flora of Kansai Basin at Lalgarh of Paschim Medinipur District in West Bengal with Special ..

\begin{tabular}{|c|c|c|c|c|}
\hline 31. & Ziziphus oenoplea (L.) Miller & $\begin{array}{l}\text { Jackal jujube/ } \\
\text { Wild Jujuba }\end{array}$ & Kankul & Rhamnaceae \\
\hline 32. & Capparis sepiaria L. & Caper bush & Kaliakra & Capparaceae \\
\hline 33. & Cocculus hirsutus (L.) Diels & Broom Creeper & Dadaya/Huyer & Menispermaceae \\
\hline 34. & $\begin{array}{l}\text { Caesalpinia bonducella (L.) } \\
\text { Fleming } \\
=\text { C. bonduc (L.) Roxb. }\end{array}$ & $\begin{array}{l}\text { Nickernut/ } \\
\text { Fever Nut/Physic Nut }\end{array}$ & Nata & Caesalpiniaceae \\
\hline 35. & Lantana camara L. & Yellow sage & Kutus & Lamiaceae \\
\hline 36. & $\begin{array}{l}\text { Dentella repens (L.) J.R.Forst \& G. } \\
\text { Forst }\end{array}$ & Creeping lick stoop & Bhumipat phool & Rubiaceae \\
\hline 37. & Ludwigia perennis L. & $\begin{array}{l}\text { Cyllindric fruit primrose- } \\
\text { willow }\end{array}$ & $\begin{array}{l}\text { Jal labanga/Ban } \\
\text { labanga }\end{array}$ & Onagraceae \\
\hline 38. & Ocimum americanum L. & Hoary basil & Ban tulsi & $\begin{array}{l}\text { Lamiaceae/ } \\
\text { Labiatae }\end{array}$ \\
\hline 39. & Ficus benghalensis L. & Banyan tree & Bot & Moraceae \\
\hline 40. & Jatropha gossypifolia L. & $\begin{array}{l}\text { Black physic nut/ } \\
\text { Bellyache bush/ } \\
\text { Cotton-leaf physicnut }\end{array}$ & Bharenda & Euphorbiaceae \\
\hline 41. & Coccinia grandis (L.) Voigt. & $\begin{array}{l}\text { Ivy gourd/ } \\
\text { Scarlet gourd }\end{array}$ & Tite Kundri & Cucurbitaceae \\
\hline 42. & Tephrosia purpurea (L.) Pers. & Wild Indigo & $\begin{array}{l}\text { Ban Nil/Lahamori } \\
\text { /Sarpunkha }\end{array}$ & Fabaceae \\
\hline 43. & $\begin{array}{l}\text { Tiliacora acuminata (Lam.) Hook. } \\
\text { f. \& Thoms. }\end{array}$ & Teliakoara & Teli lata & Menispermaceae \\
\hline 44. & $\begin{array}{l}\text { Chrysopogon aciculatus (Retz.) } \\
\text { Trin. }\end{array}$ & $\begin{array}{l}\text { Mackie's pest/ } \\
\text { Lesser Spear grass }\end{array}$ & Chorkanta & Poaceae \\
\hline 45. & Tragia hispida Willd. & Stinging Neetle & Bichuti & Euphorbiaceae \\
\hline 46. & Martynia annua L. & Tiger's Claw & Baghnokh & $\begin{array}{l}\text { Martyniaceae } \\
\text { /Pedaliaceae }\end{array}$ \\
\hline 47. & Calotropis gigantean (L.) Ait. & Madar/Swallow wort & Akanda & Asclepiadaceae \\
\hline 48. & $\begin{array}{l}\text { Eupatorium odoratum L. } \\
=\text { Chromolaena odorata (L.) R.M. } \\
\text { King. \& H. Rob. }\end{array}$ & $\begin{array}{l}\text { Christ mas bush/ } \\
\text { Common flosh } \\
\text { flower/Siam weed/jack in } \\
\text { the bush }\end{array}$ & $\begin{array}{l}\text { Ban } \\
\text { karpur/Kaslimasala/ } \\
\text { Sial muti/Banmara }\end{array}$ & Asteraceae \\
\hline 49. & Combretum decandrum Jacq. & - & Atang & Combretaceae \\
\hline 50. & Dicliptera bupleuroides Nees & - & Lal Jhanti/lal sira & Acanthaceae \\
\hline 51. & Hyptis suaveolens (L.) Poit. & Bush tea-bush & Bilati tulsi & Lamiaceae \\
\hline 52. & $\begin{array}{l}\text { Anisomeles ovata W. T. Aiton } \\
=\text { A. indica (L.) Kuntze. }\end{array}$ & Catmint & Gopali/Gobru & Lamiaceae \\
\hline 53. & $\begin{array}{llll}\begin{array}{l}\text { Alangium } \\
\text { Wangerin }\end{array} & \text { salvifolium } & \text { (L. } & \text { f. }) \\
\end{array}$ & Sage-leaved alangium & Ankar/Ans phal & Alangiaceae \\
\hline 54. & $\begin{array}{l}\text { Holoptelea integrifolia } \text { (Roxb.) } \\
\text { Planch }\end{array}$ & Jungle cork wood tree & Nata karanja/Challa & Ulmaceae \\
\hline 55. & $\begin{array}{l}\text { Haldina cordifolia (Roxb.) Rids. } \\
=\text { Adina cordifolia (Roxb.) Benth. } \\
\text { \& Hook. f. ex B. D. Jacks. }\end{array}$ & Heart leaf Adina & Karam & Rubiaceae \\
\hline 56. & Sphaeranthus indicus L. & East Indian Globe thistle & $\begin{array}{l}\text { Chagal } \\
\text { nudi/Mundi/ } \\
\text { Gorkhmundi }\end{array}$ & Asteraceae \\
\hline 57. & Tamarindus indica L. & Tamarind & Tentul & Caesalpiniaceae \\
\hline
\end{tabular}

N.B.: = Synonym

V. Figures in Result (Fig. 1 -53)

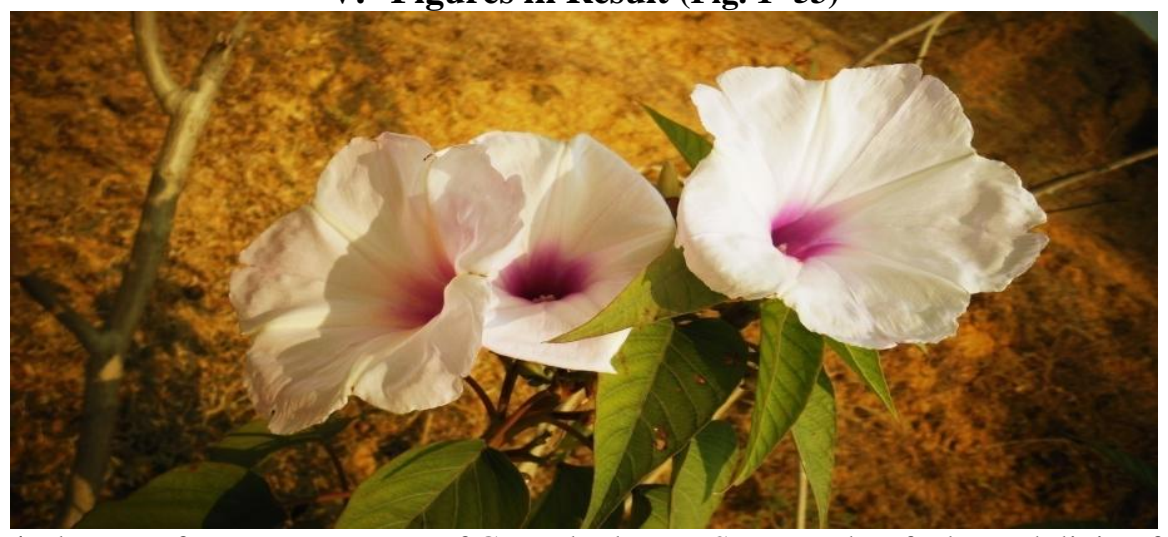

Fig. 1 Flowers of Ipomoea carnea of Convolvulaceae-Stem used as fuel wood, living fence 


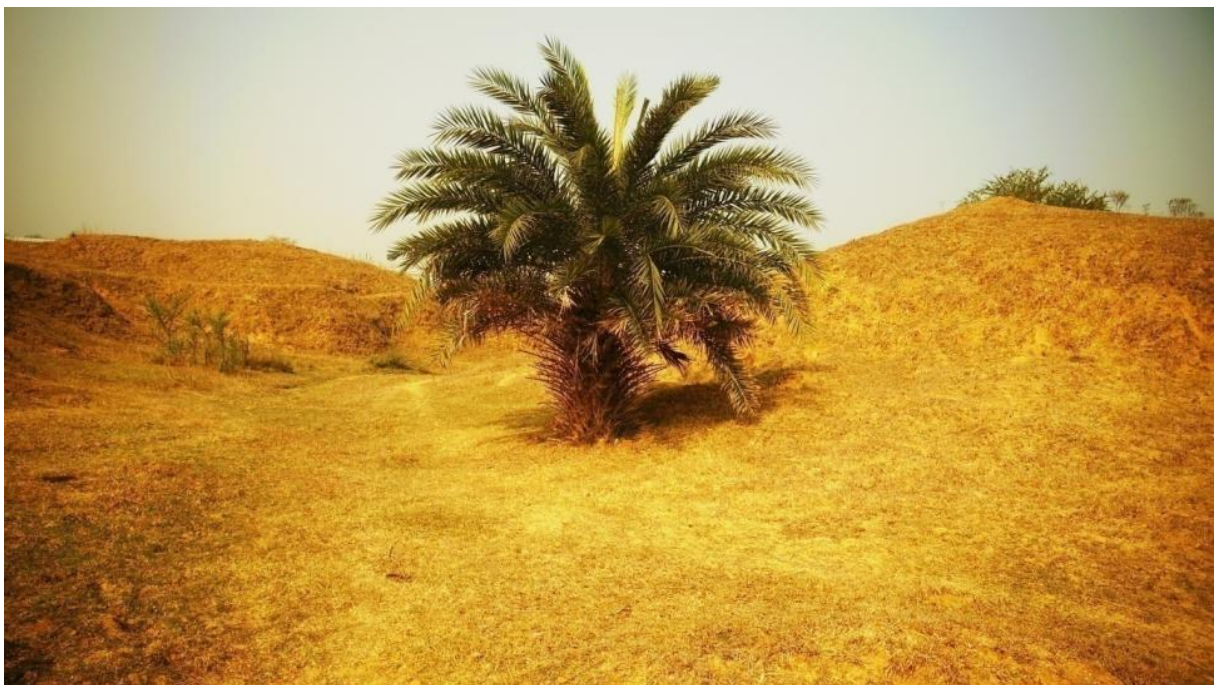

Fig. 2 Phoenix sylvestris of Arecaceae (Eng.-Date palm) is under threat need immediate conservation. The plant is toddy making and molasses yielding plant also get edible fruits

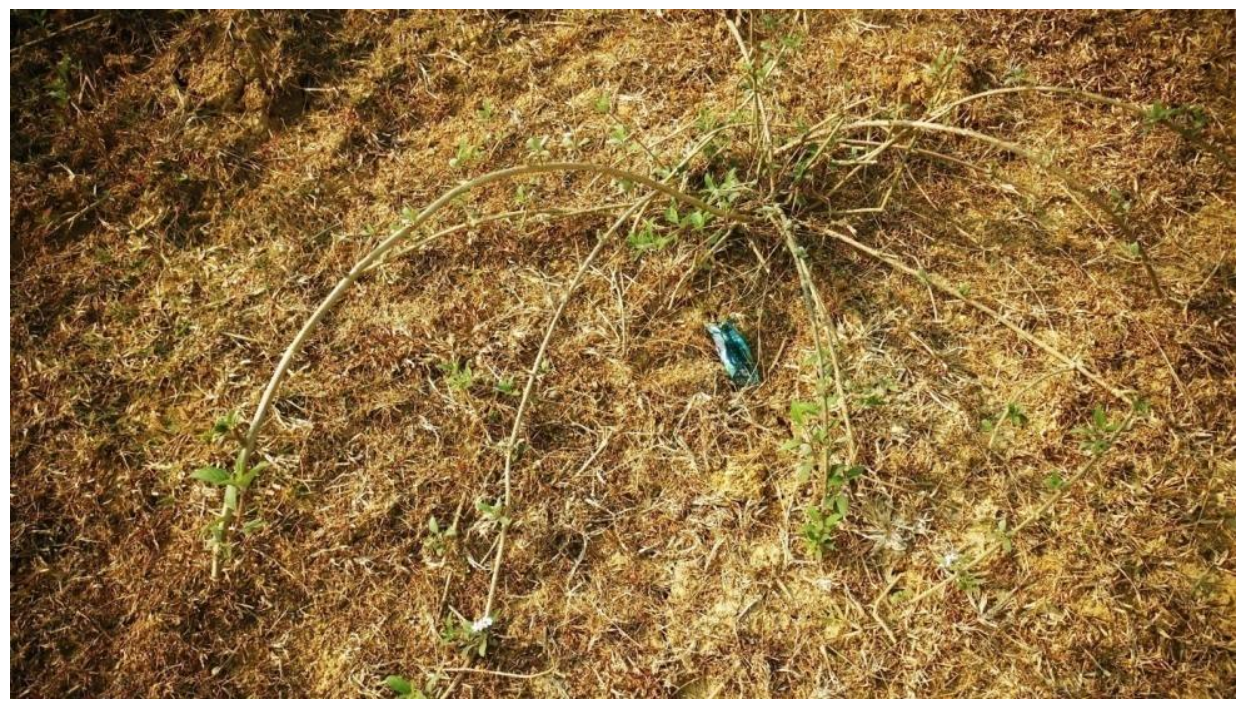

Fig. 3 Lippia geminata of Verbenaceae-a weed of River basin at Lalgarh, Paschim Medinipur

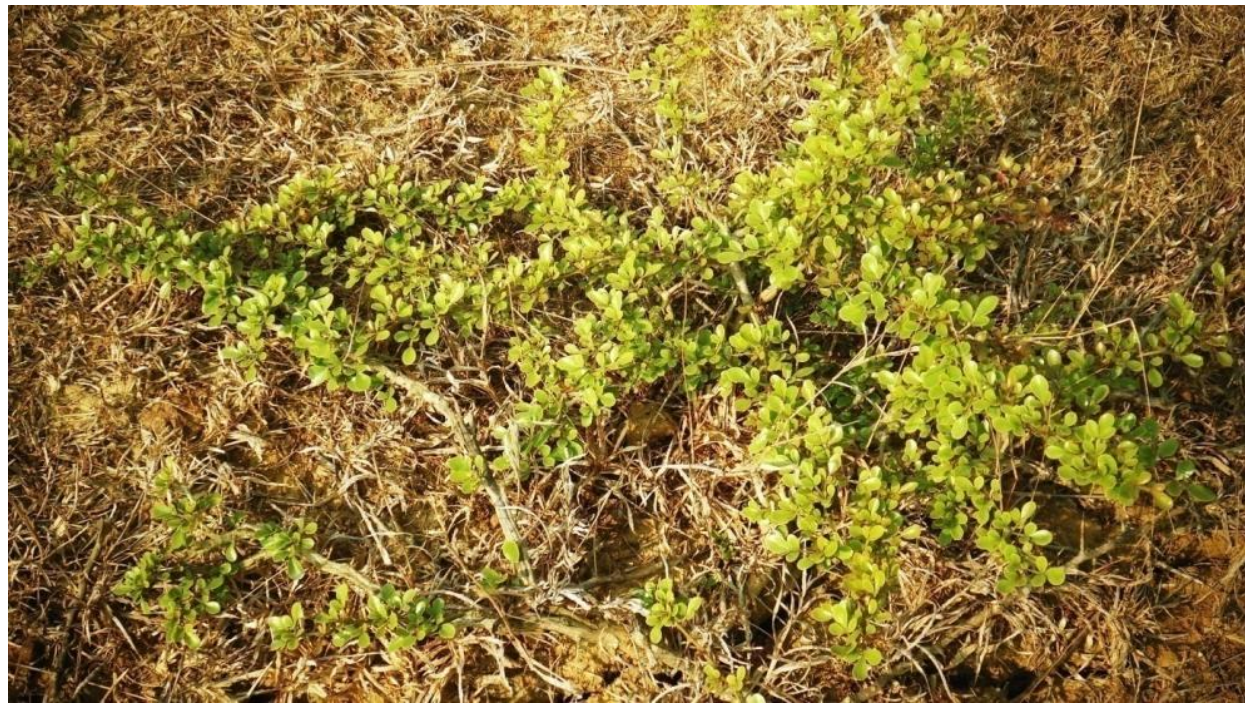

Fig. 4 Feronia elephantum Correa of Rutaceae yield edible fruits used to prepare chutney (young plant) 


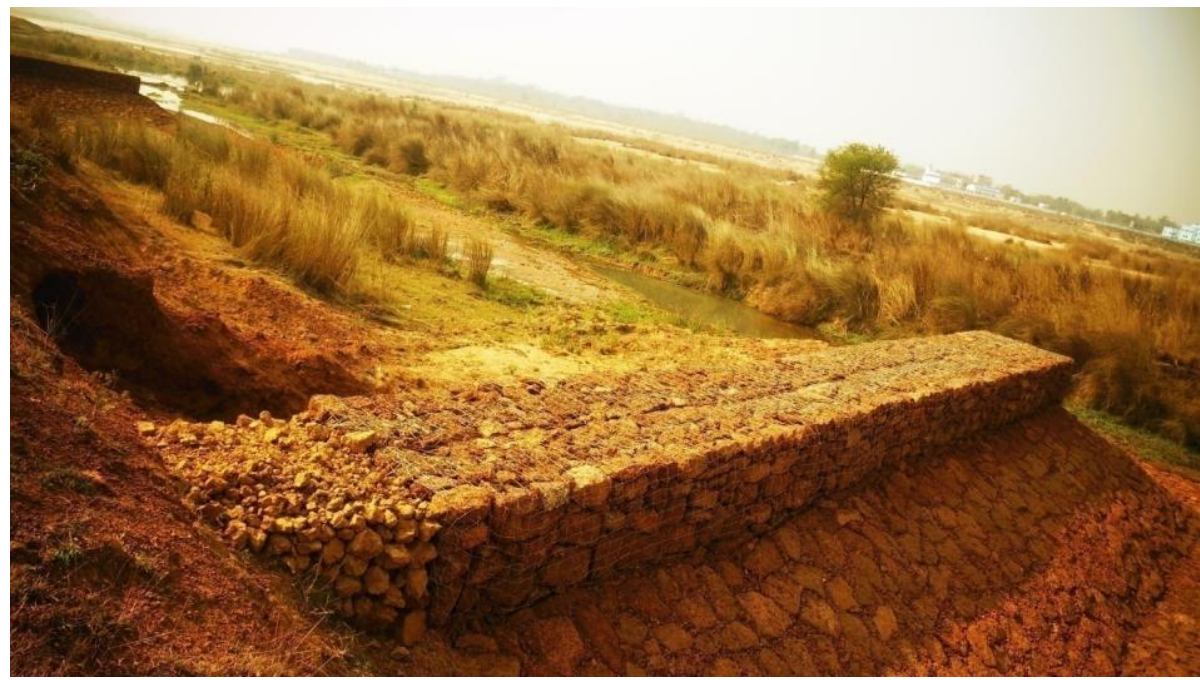

Fig. 5 Huge hedge (Kash-Saccharum spontaneum L.) in river basin and Acacia a common vegetation spectrum along the basin of Kansai

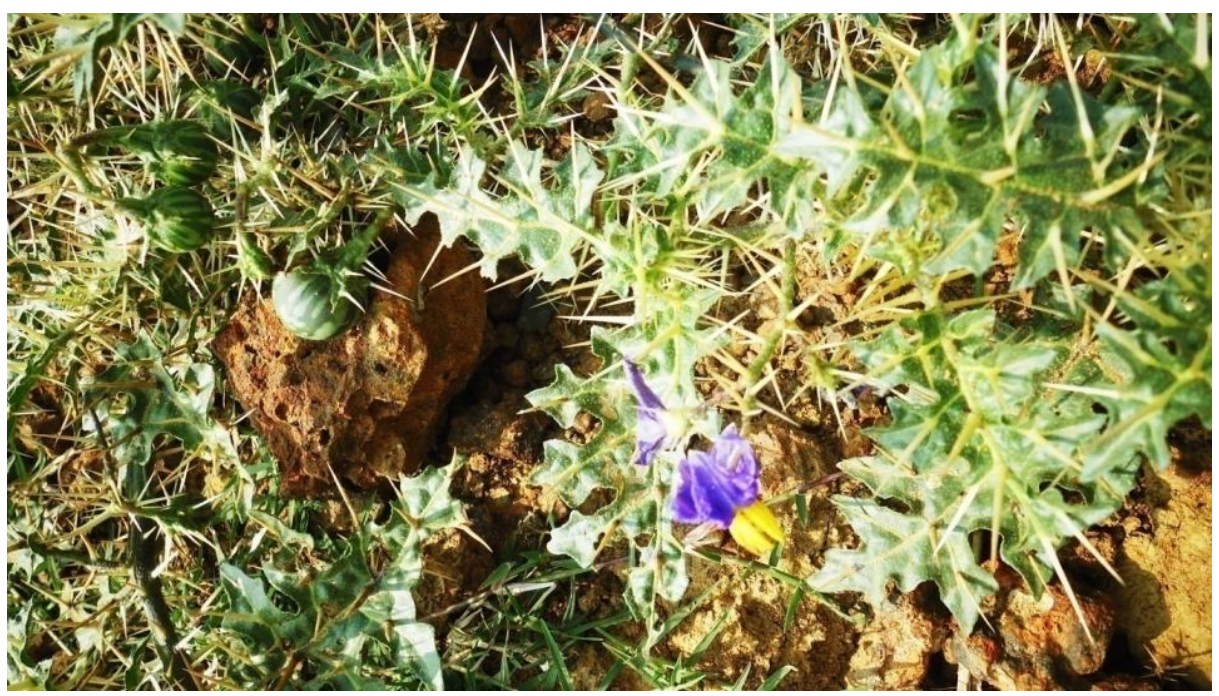

Fig. 6 Solanum xanthocarpum of Solanaceae showing fruits and flowers an important medicinal plant

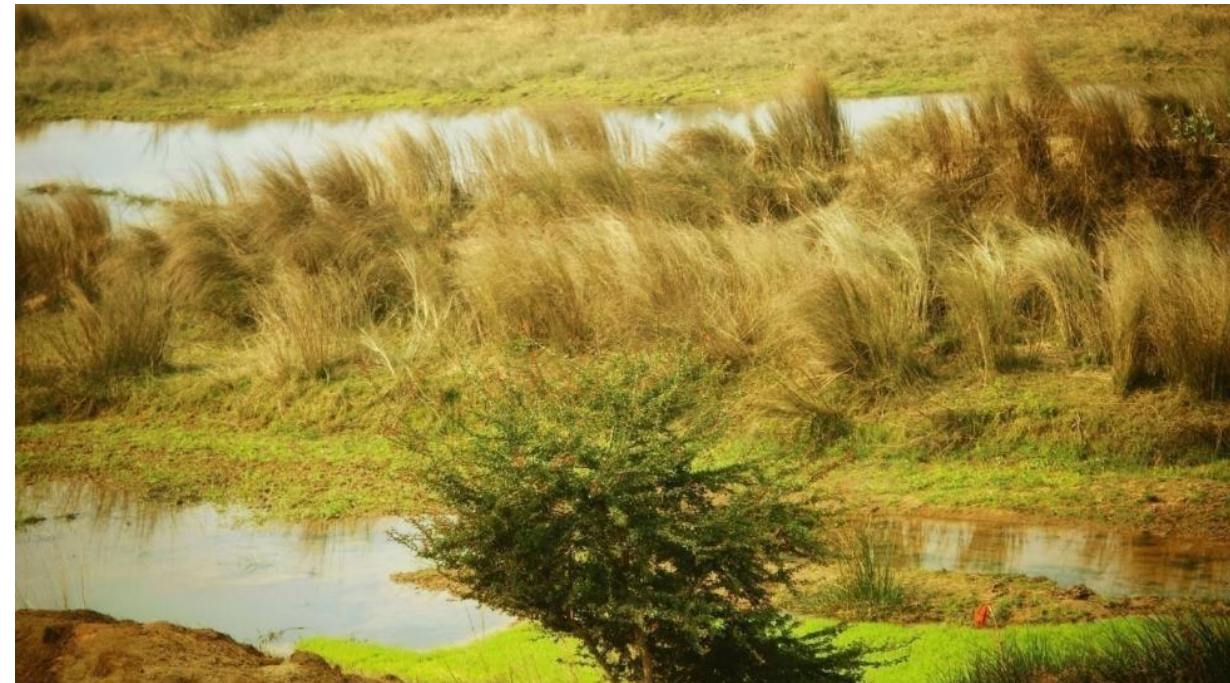

Fig. 7 Paddy seedlings at Kansai basin behind the Pithecellobium dulce (Mimosaceae) plant during winter before shifting in a paddy field. 
Flora of Kansai Basin at Lalgarh of Paschim Medinipur District in West Bengal with Special ..

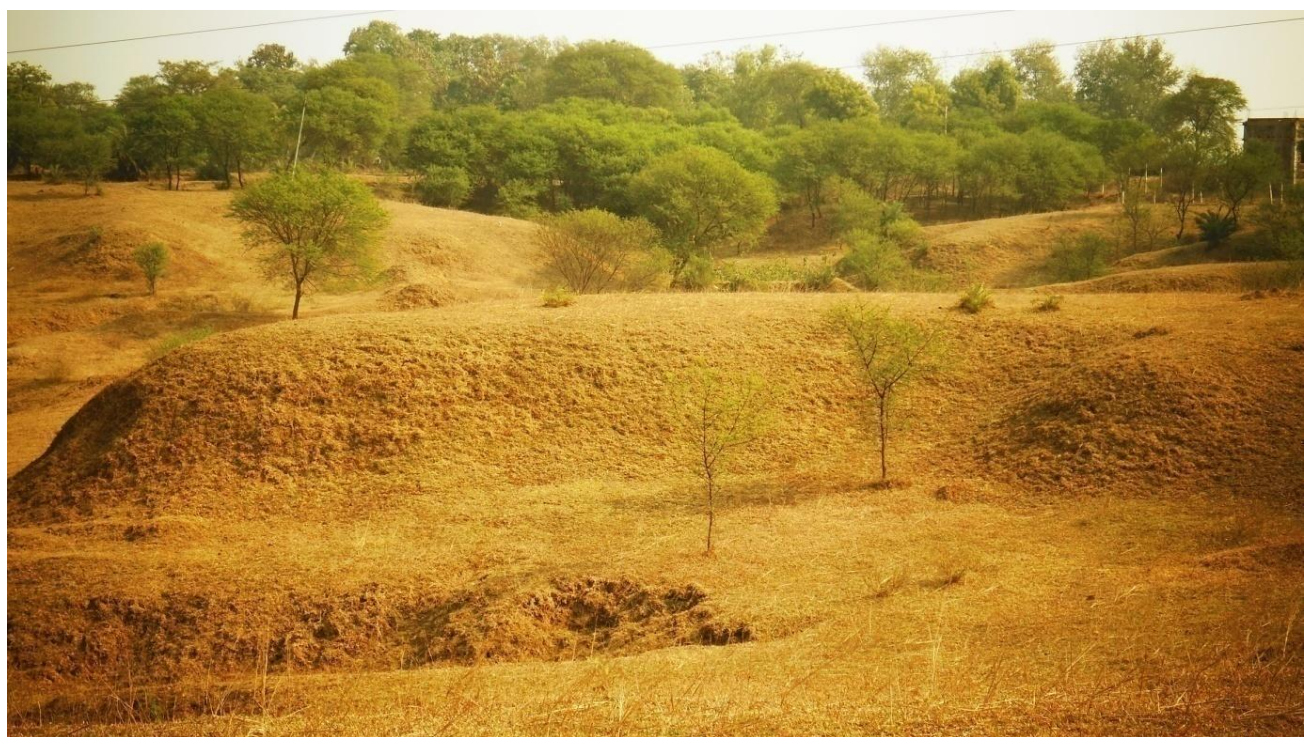

Fig. 8 Vegetation of degraded land filled with Acacaia nilotica trees; note that the land is undulated

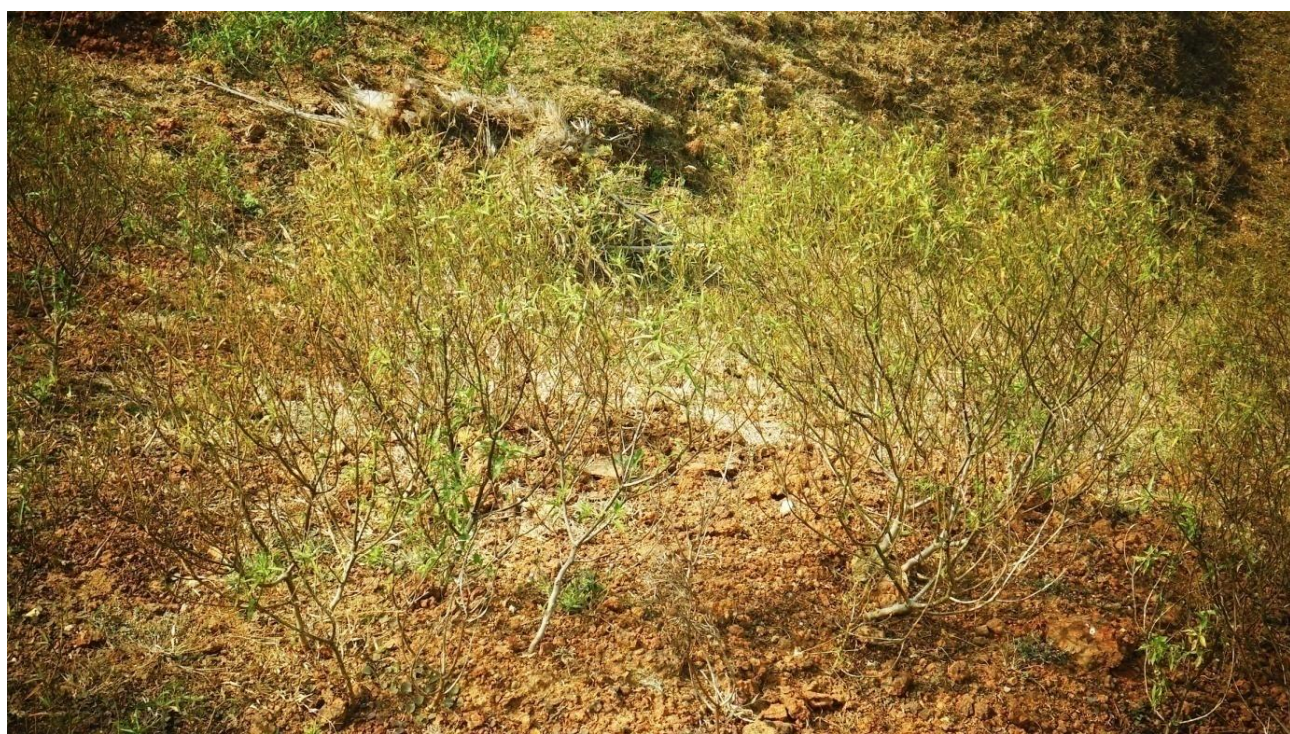

Fig. 9 Croton bonplandianum of Euphorbiaceae-A common medicinal plant used to treat the toothache.

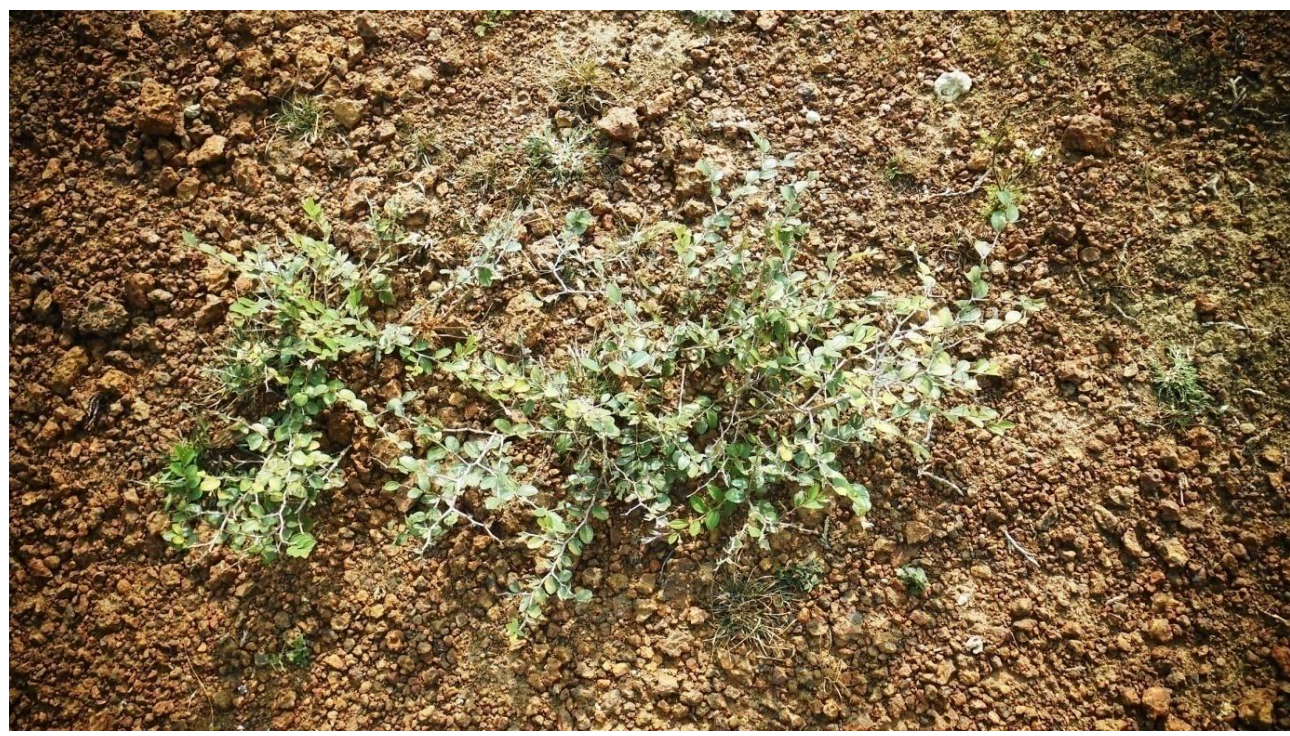

Fig. 10 Ziziphus sp. of Rhamnaceae in degraded land of Kansai river bank at Lalgarh 


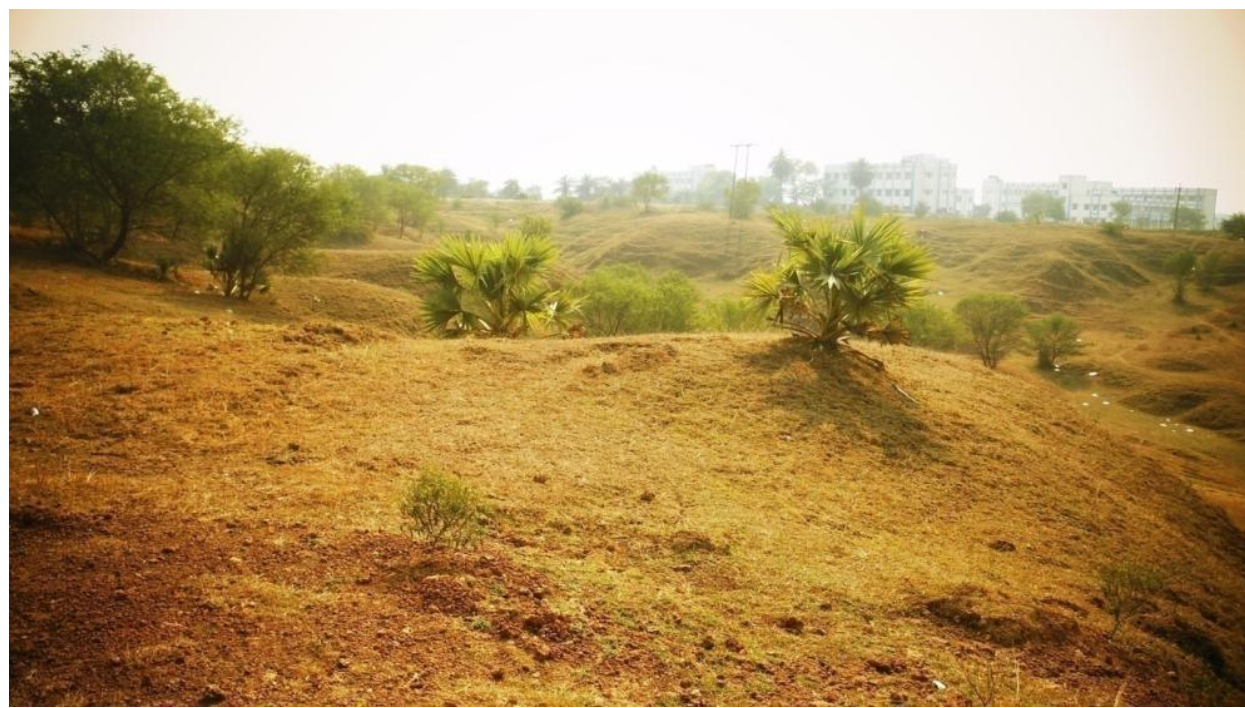

Fig 11 Borassus flabellifer of Arecaceae an important economic plant used to prepare molasses and toddy, note that the plant is under threat need conservation

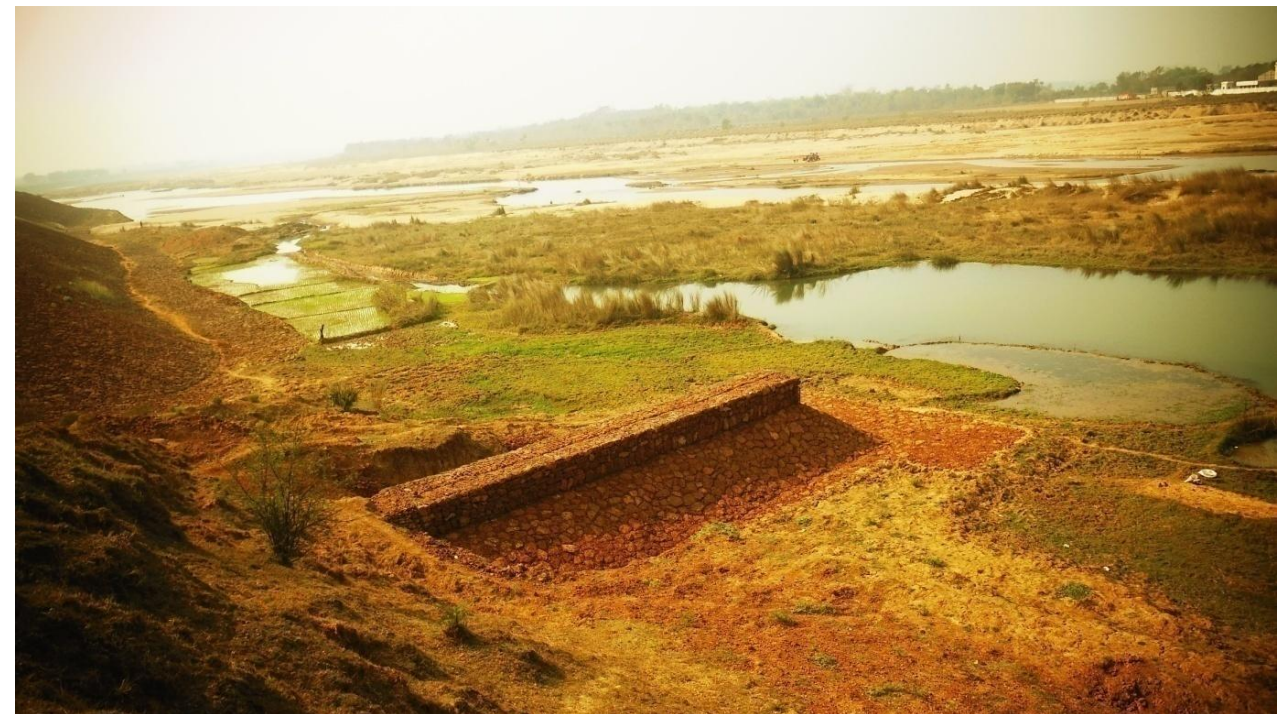

Fig. 12 Paddy/Rice field (Oryza sativa L.) in Kansai basin at Lalgarh of Paschim Medinipur

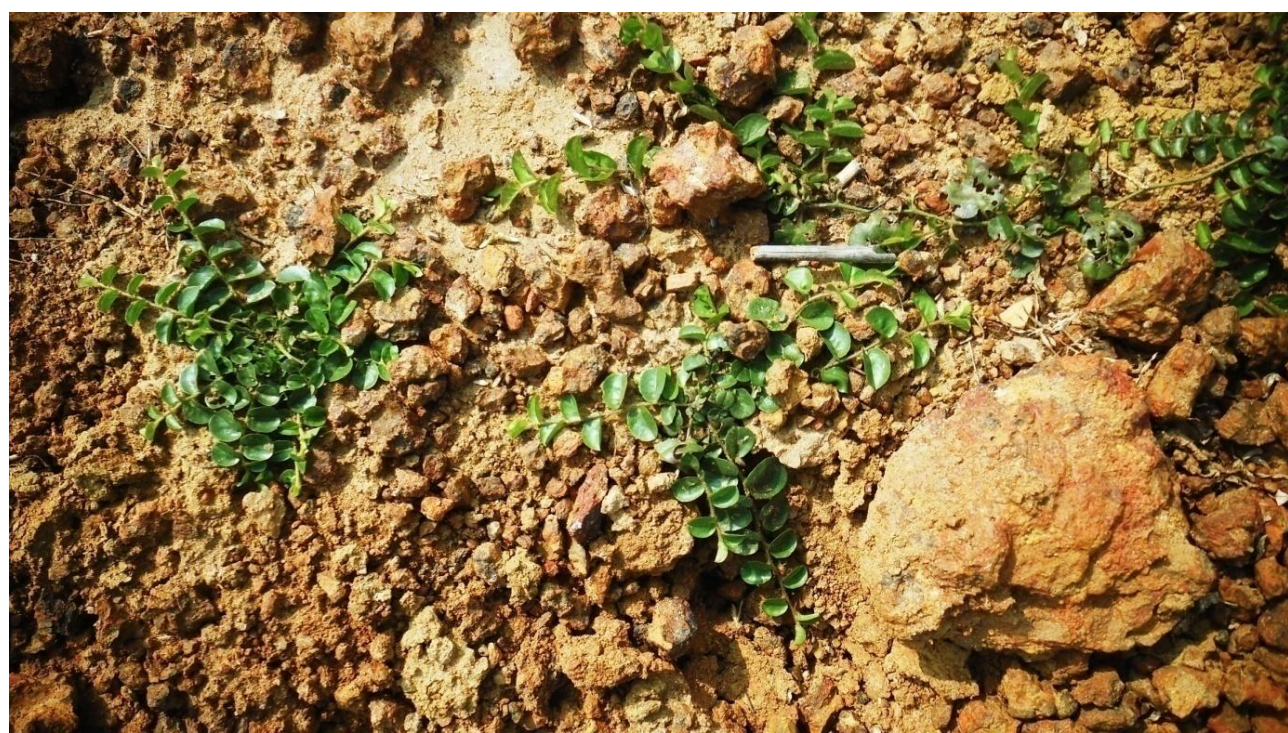

Fig. 13 Evolvulus nummularius L. of Convolvulaceae-a medicinal plant 
Flora of Kansai Basin at Lalgarh of Paschim Medinipur District in West Bengal with Special ..

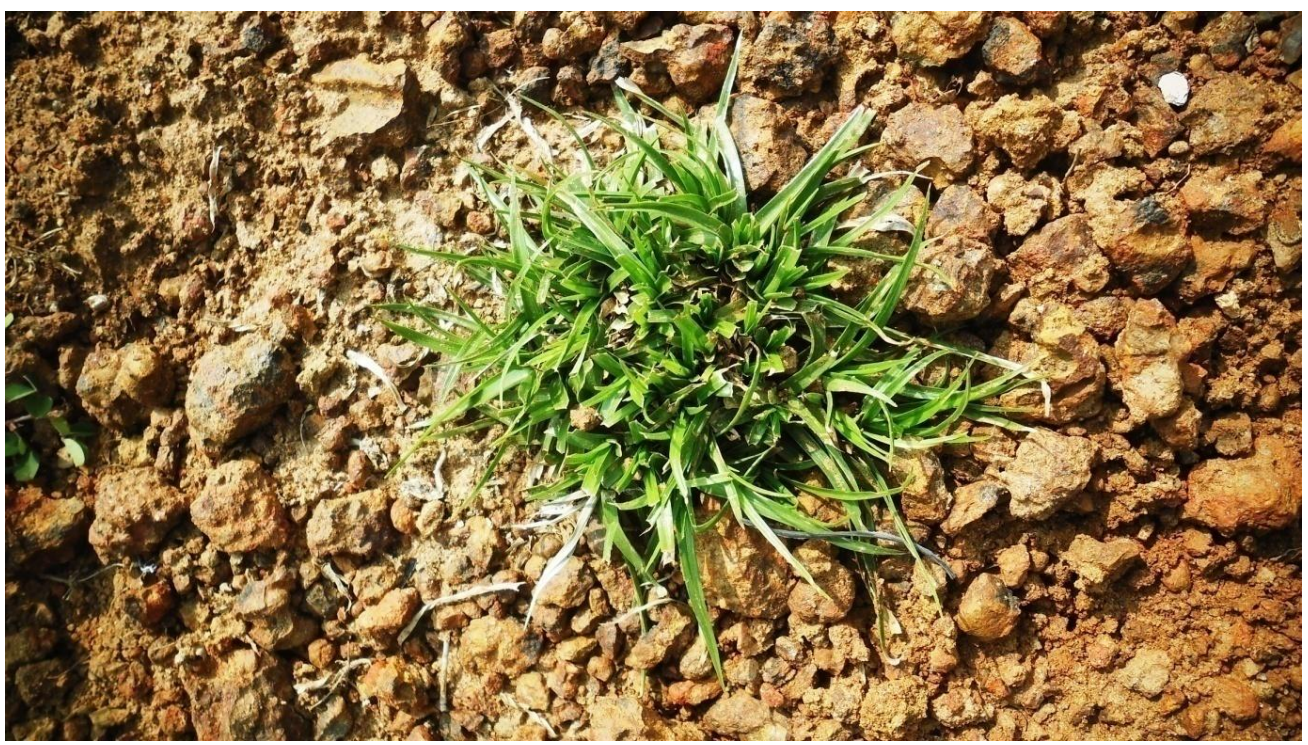

Fig. 14 Cyperus rotundus L. of Cyperaceae-a sedge of river bank Kansai

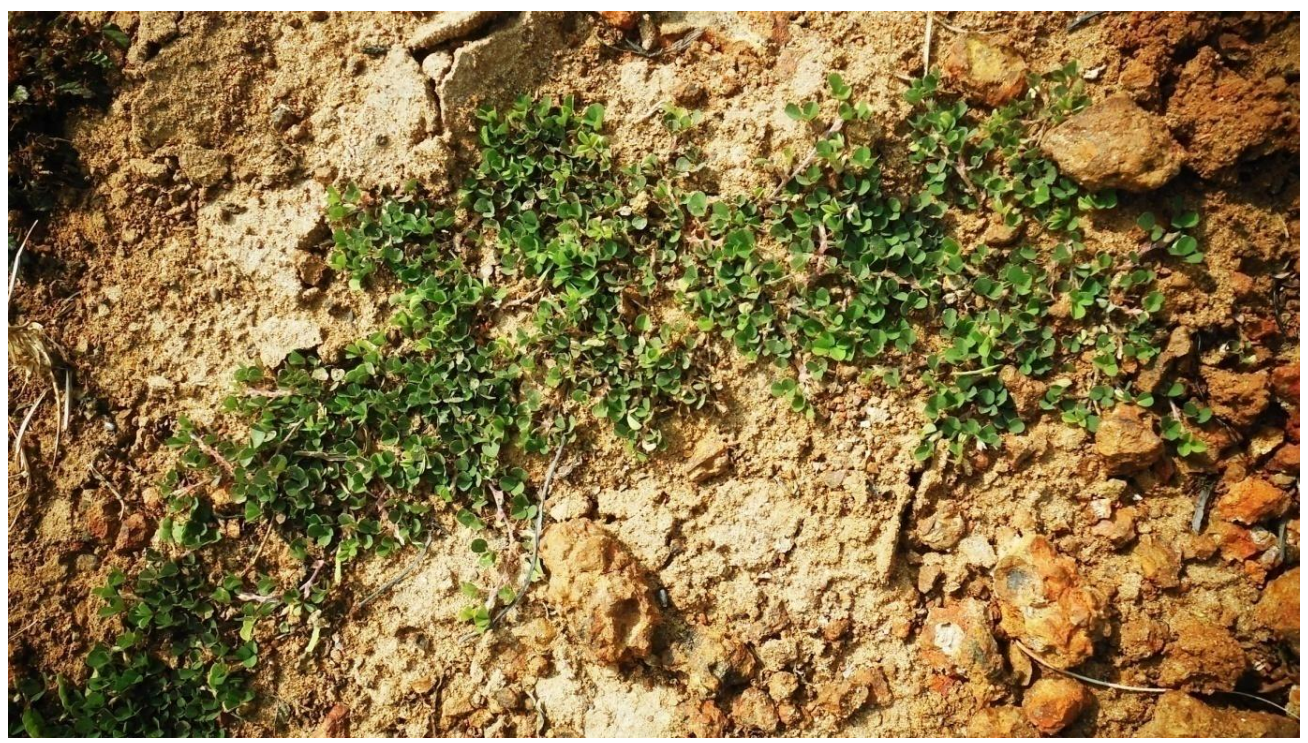

Fig. 15 Desmodium triflorum (L.) DC. of Fabaceae-A weed and medicinal plant is a source of Rhizobium sp.

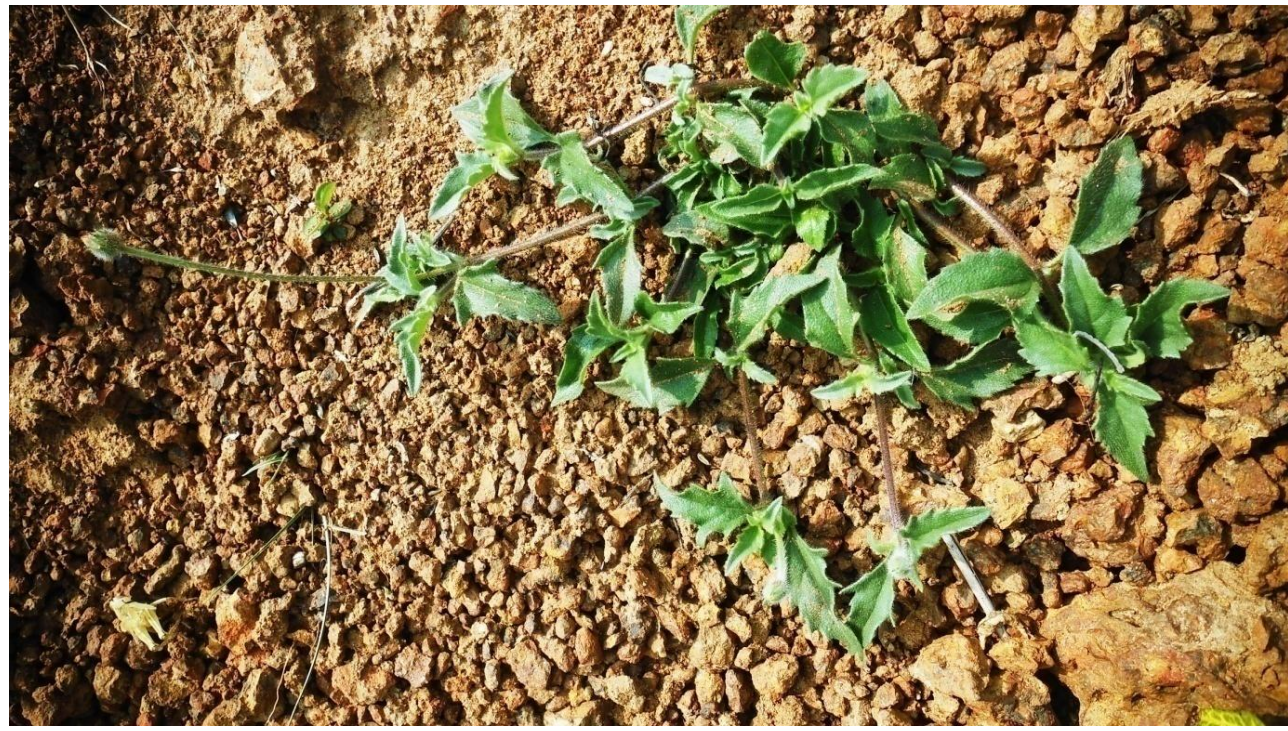

Fig. 16 Tridax procumbens L. of Asteracea is a medicinal plant, leaf decoction is used to stop bleeding 
Flora of Kansai Basin at Lalgarh of Paschim Medinipur District in West Bengal with Special ..

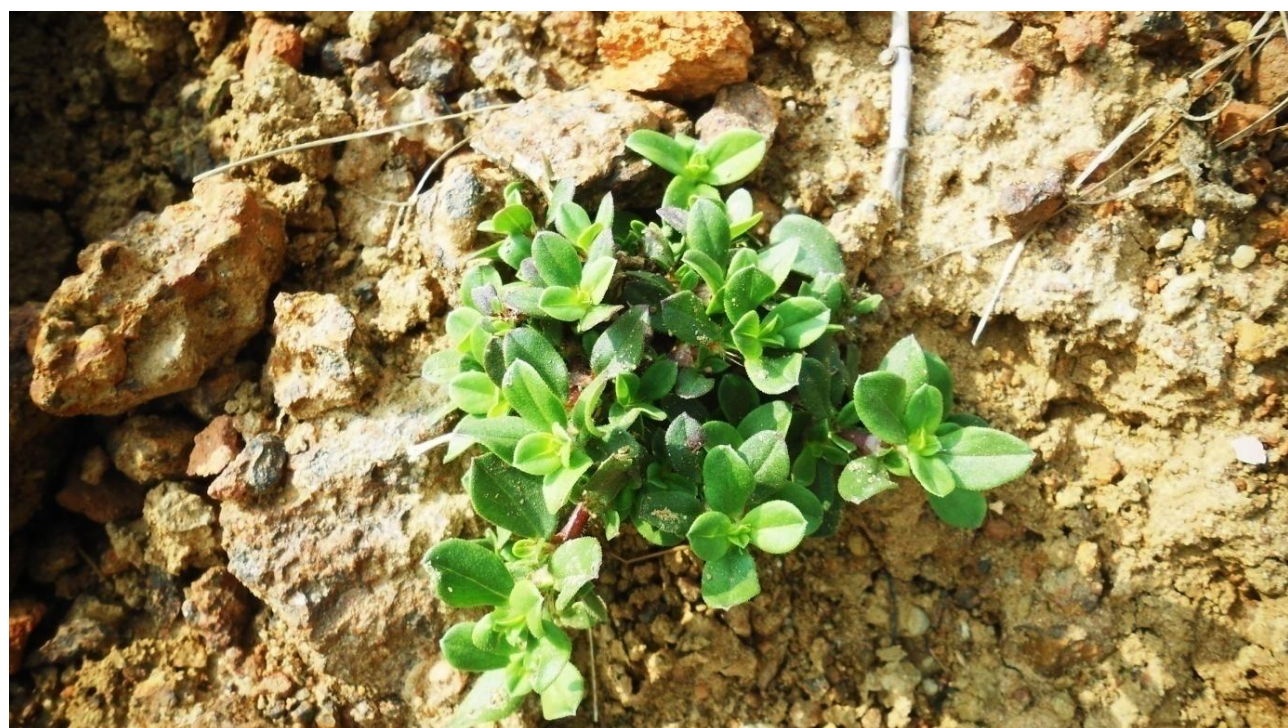

Fig. 17 Spermacoce hispida L. of Rubiaceae during winter at Kansai river bank of Lalgarh

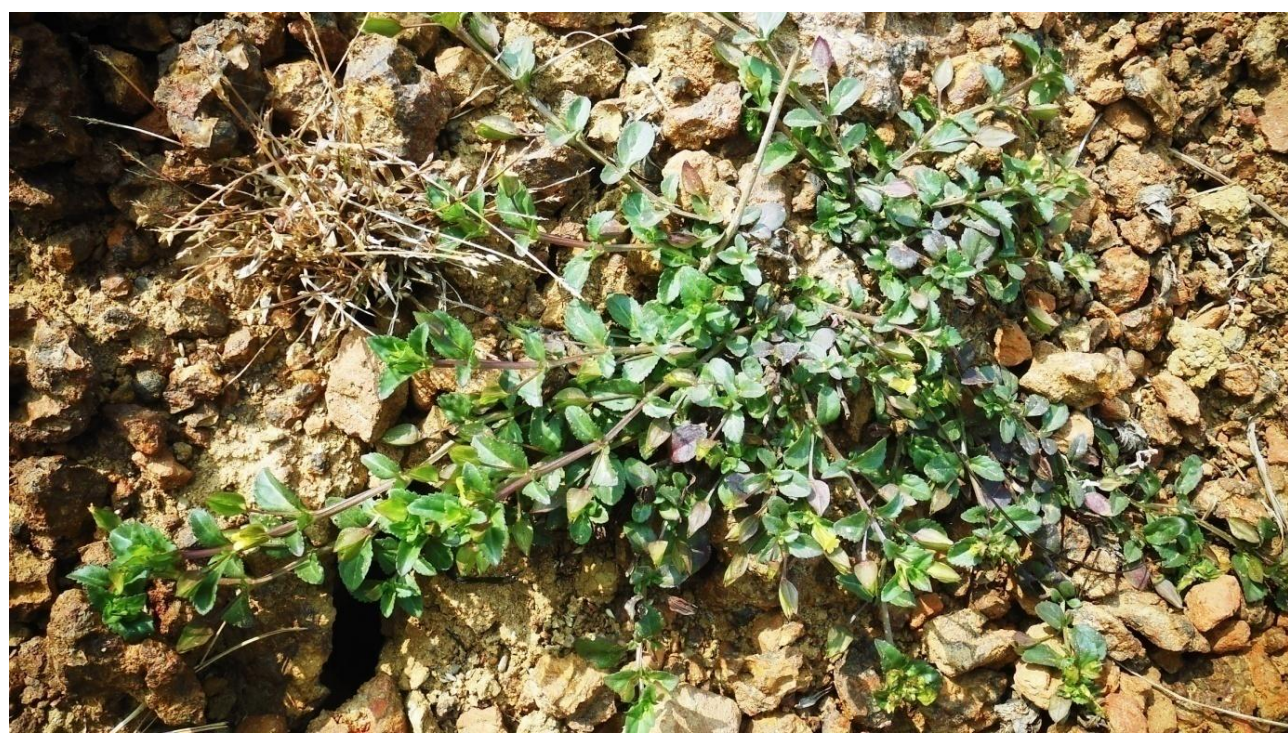

Fig. 18 Mecardonia procumbens (Mill.) Small of Scrophulariaceae-a medicinal plant is called Gorur Brmhi

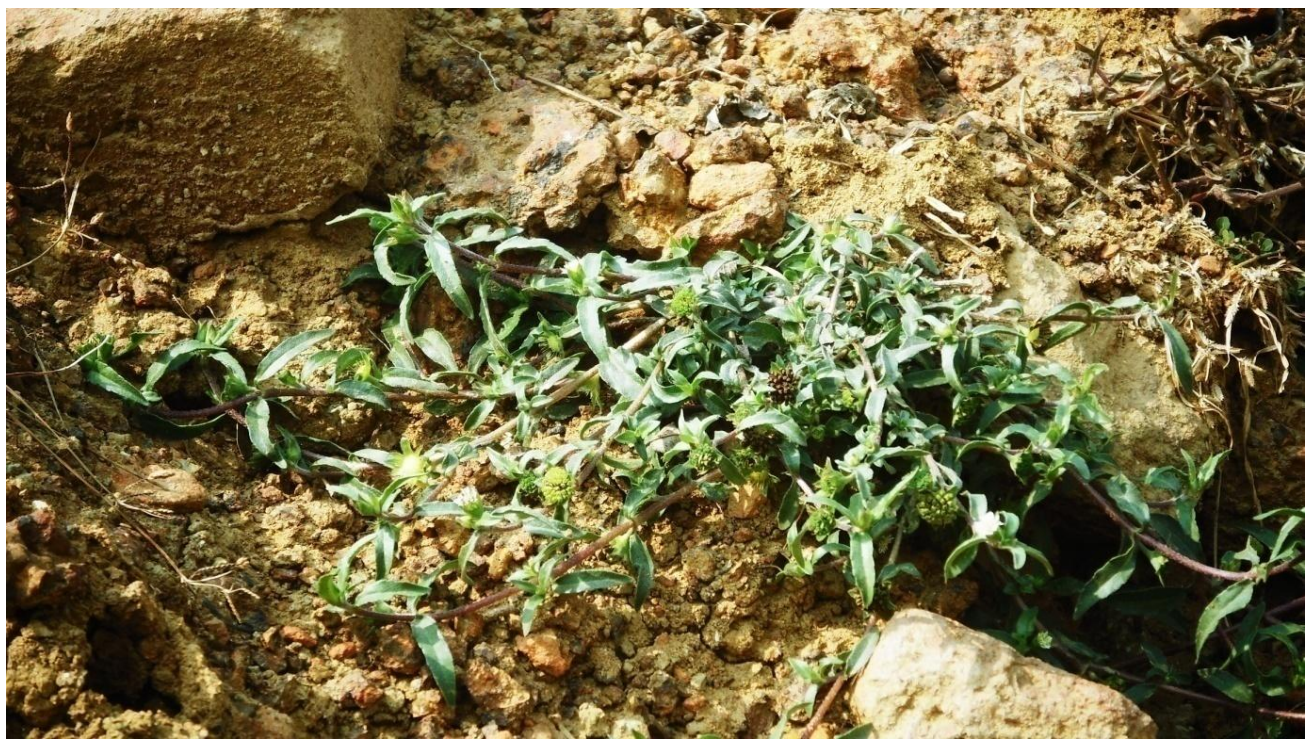

Fig. 19 Eclipta prostrata L. of Asteraceae-an important medicinal herb also used to prepare dye 
Flora of Kansai Basin at Lalgarh of Paschim Medinipur District in West Bengal with Special ..

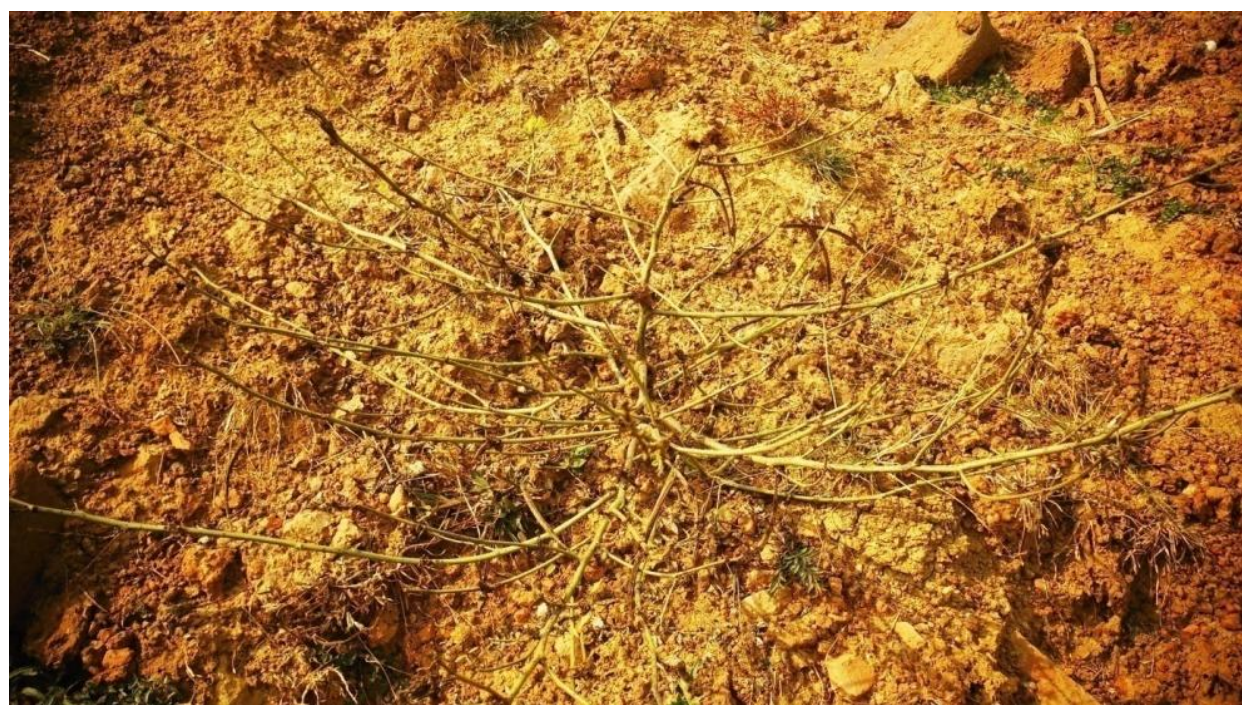

Fig. 20 Casssia tora L. of Caesalpiniaceae-A medicinal plant and also is a source of Rhizobium sp.

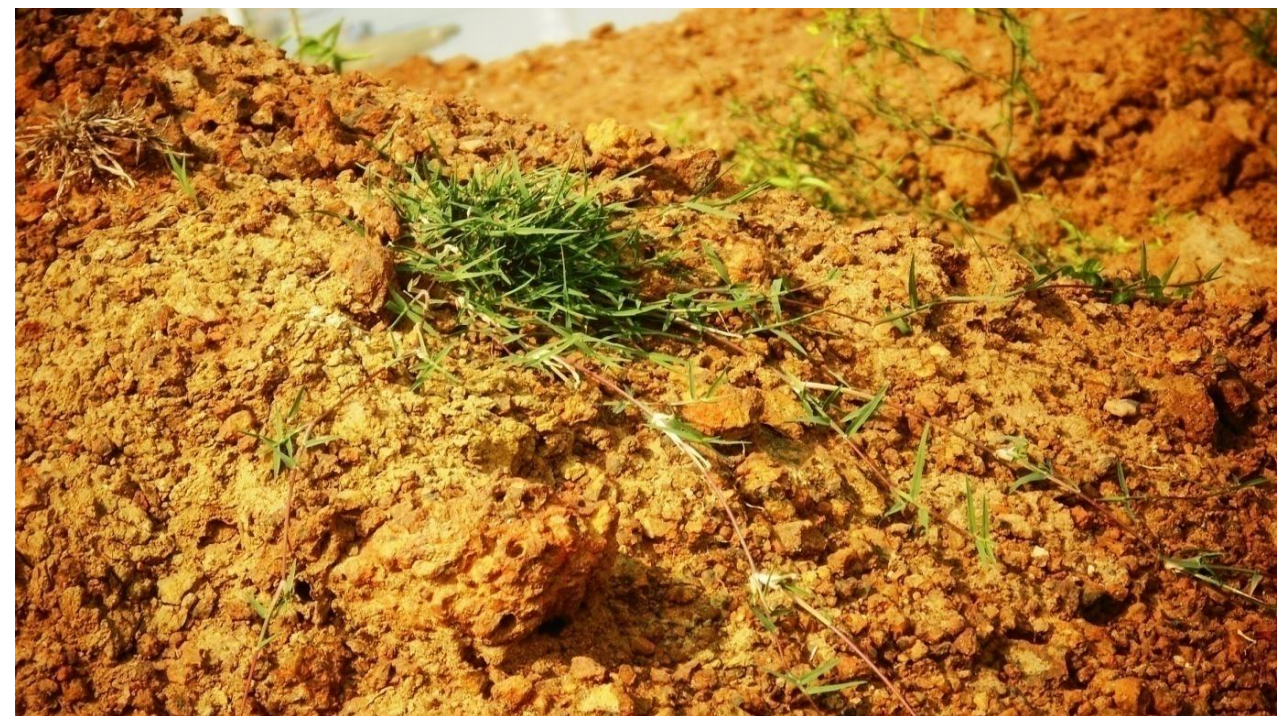

Fig. 21 Dove grass i.e. Cynodon dactylon (L.) Pers. of Poaceae is a fodder grass and also a medicinal plant

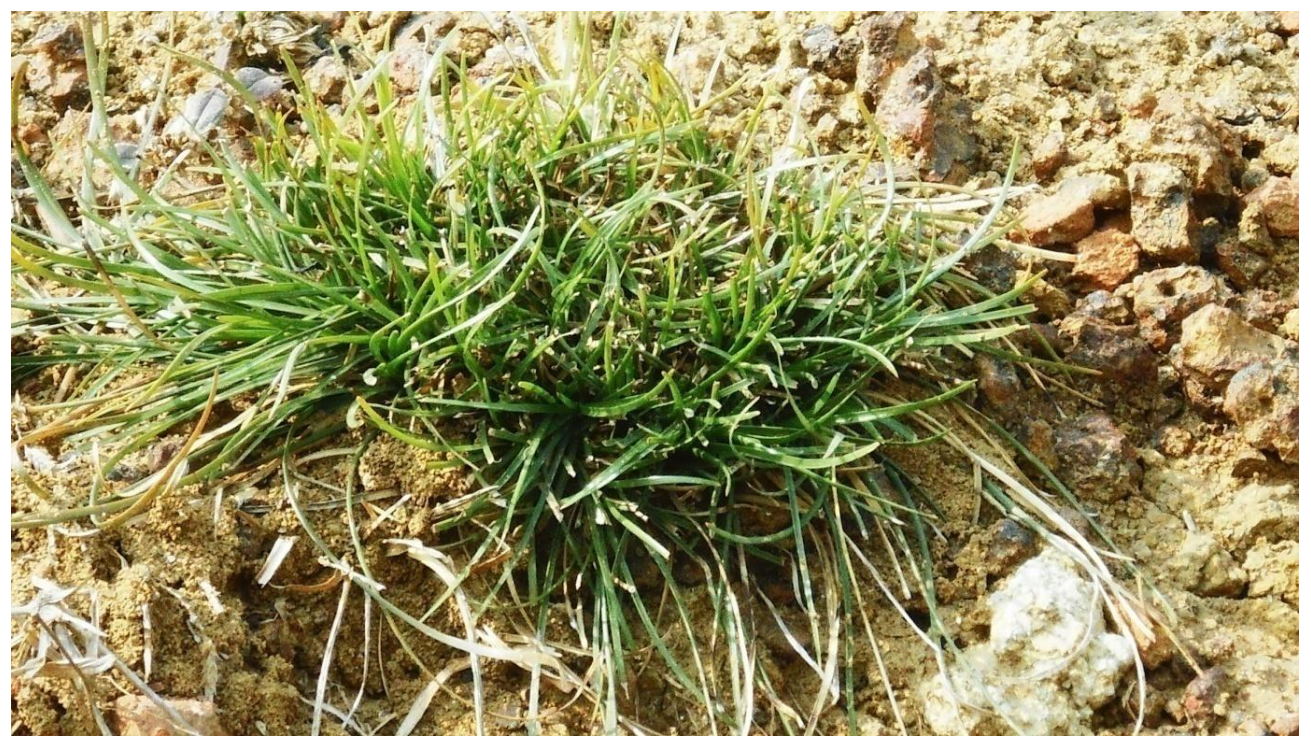

Fig. 22 Kyllinga brevifolia Rottb. of Cyperaceae is a perennial herb and is used as soil binder, fodder etc. 
Flora of Kansai Basin at Lalgarh of Paschim Medinipur District in West Bengal with Special ..

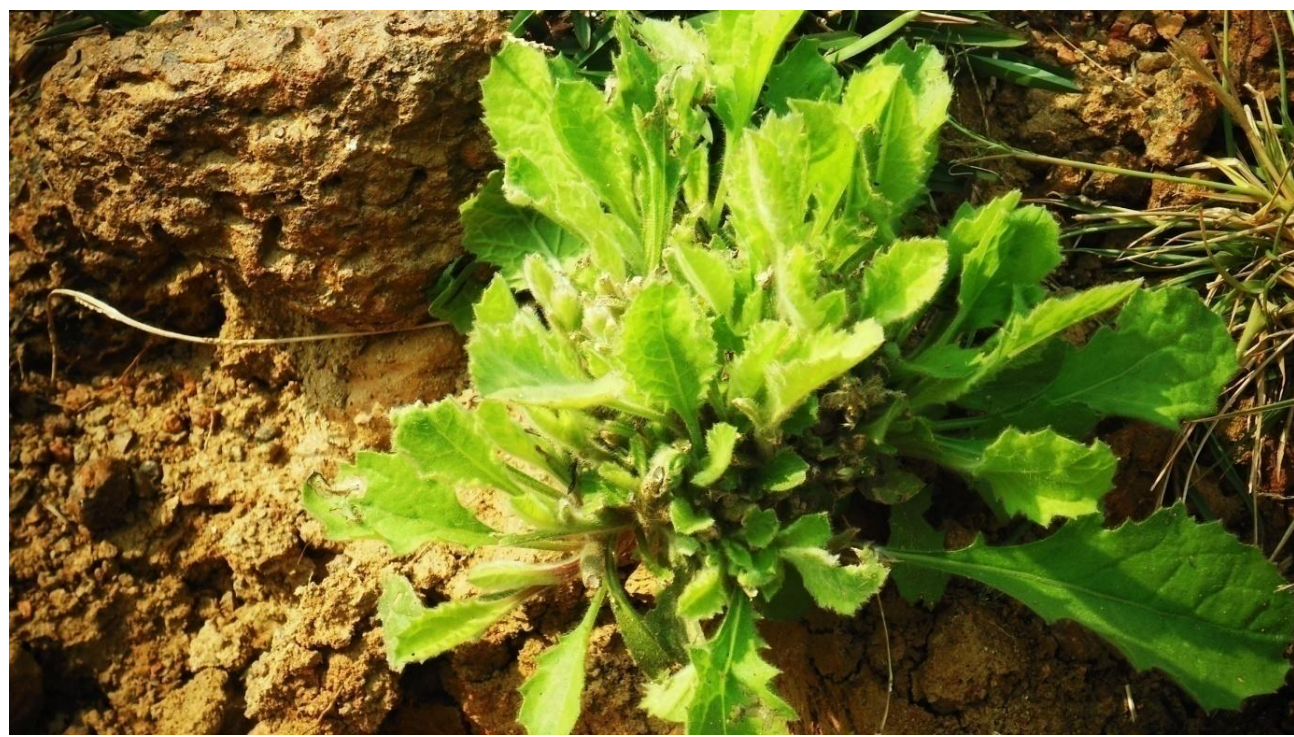

Fig. 23 Blumea oxyodonta DC. of Asteraceae is a medicinal plant, leaf decoction used to stop bleeding

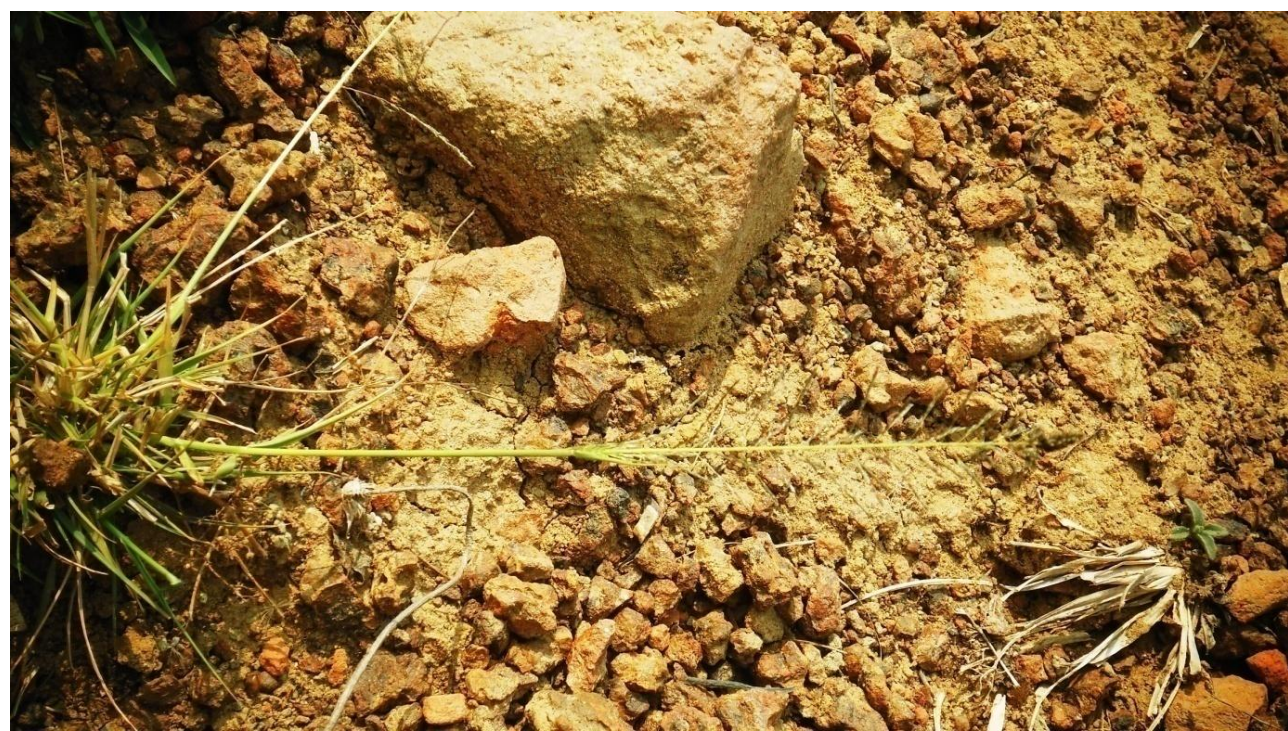

Fig. 24 Eragrostis tenella (A. Rich.) Hochst. ex Steud of Poaceae- an important fodder plant

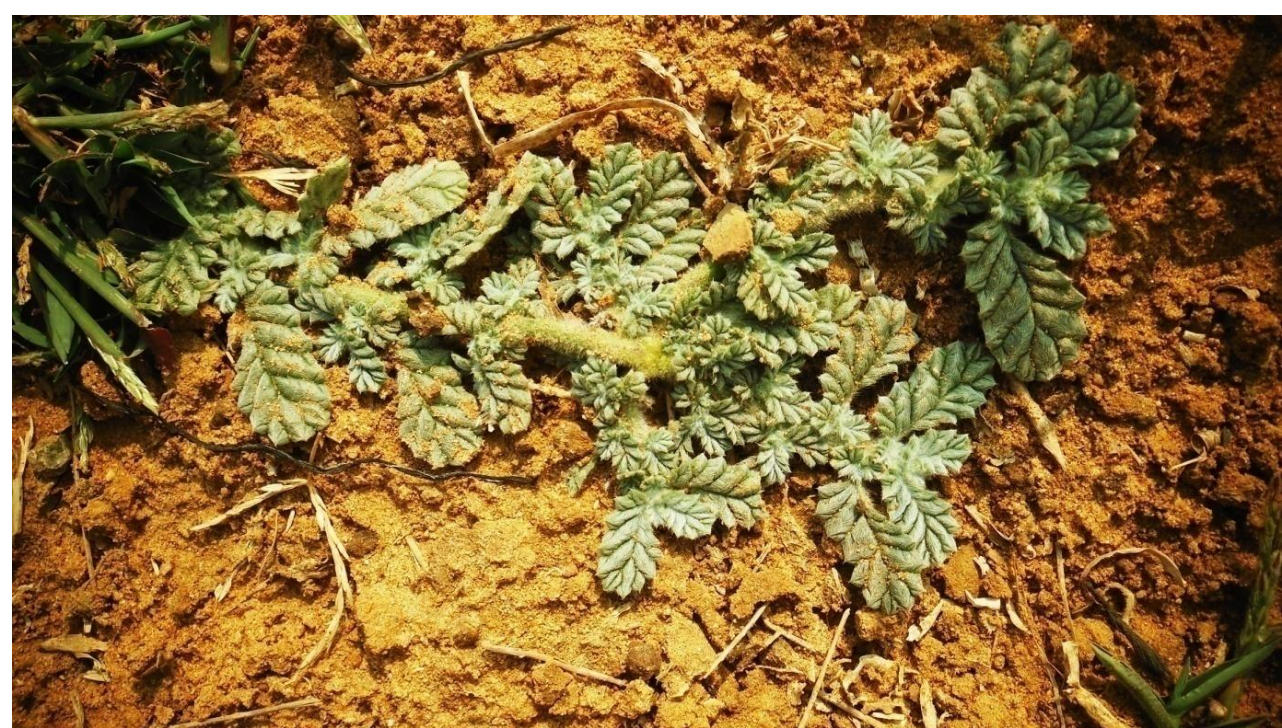

Fig. 25 Coldenia procumbens L. of Ehretiaceae (=Boraginaceae/Cordiaceae) -an anticancer plant 


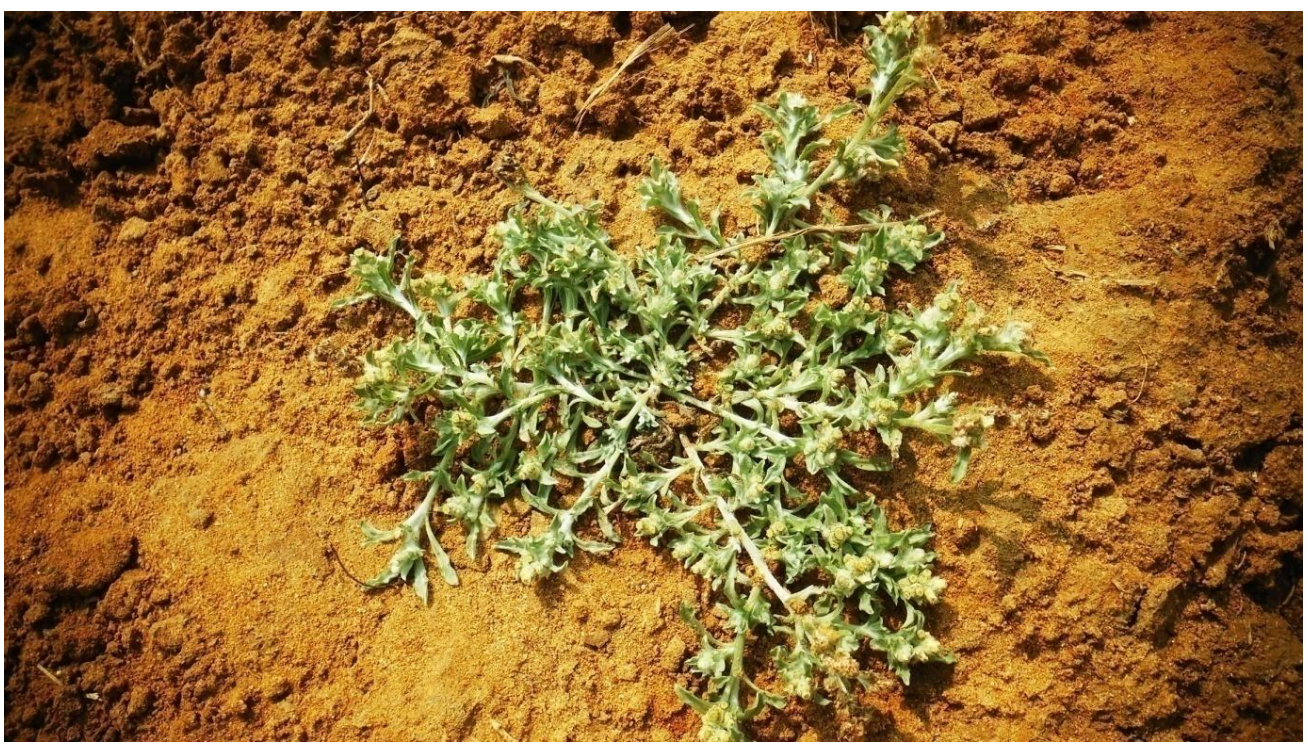

Fig. 26 Gnaphalium luteoalbum L. of Asteraceae in river bank of Lalgarh

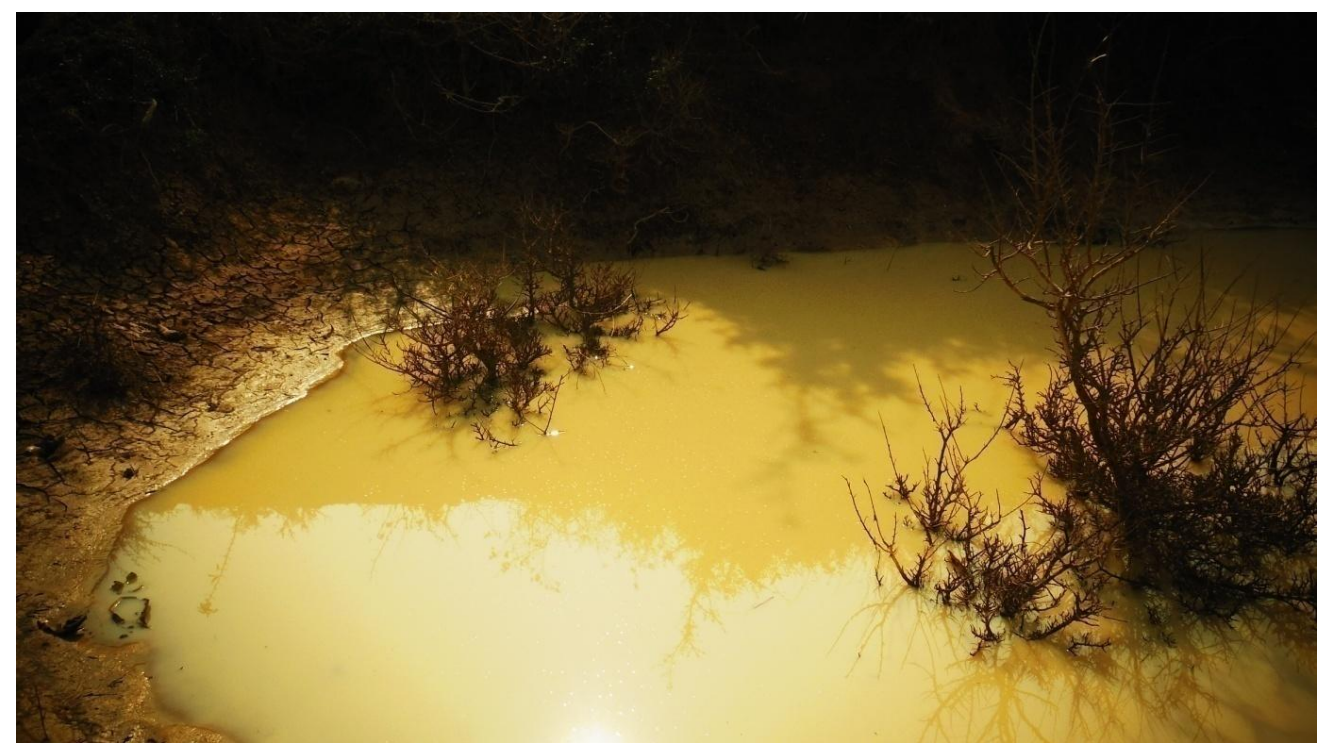

Fig. 27 A water body near river bank with sub-merged Streblus asper Lour. Plant

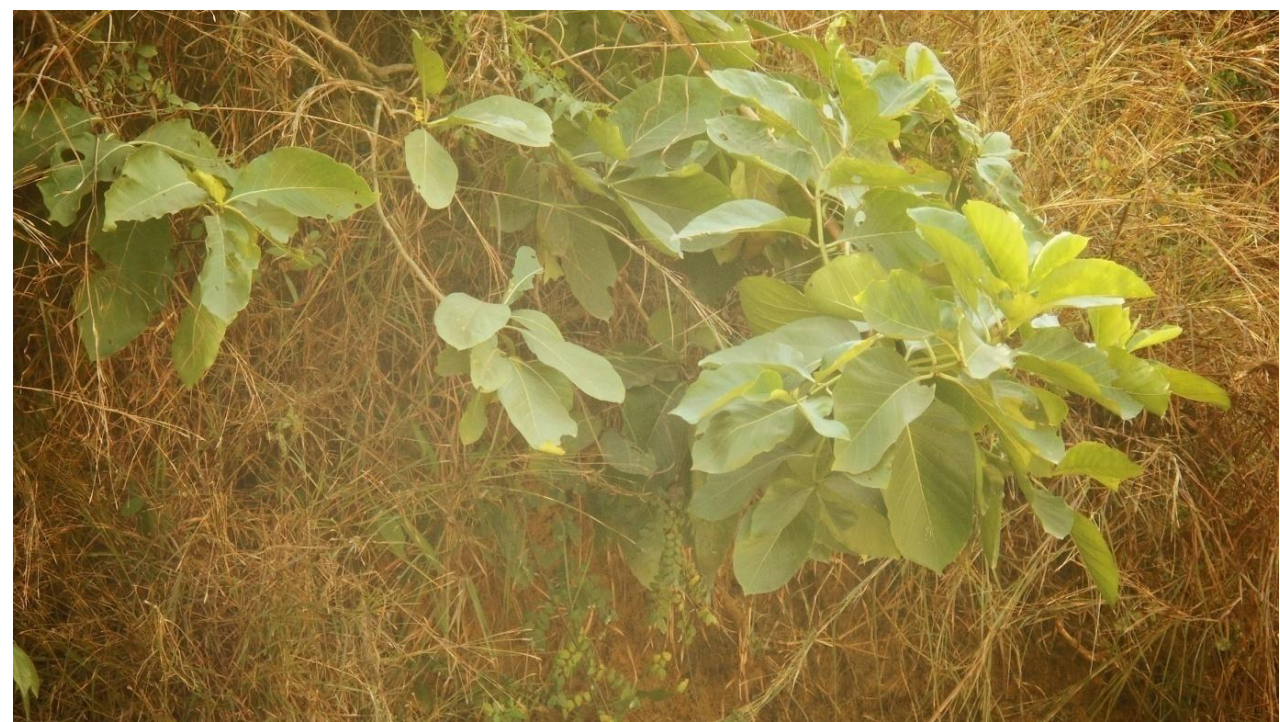

Fig. 28 Morinda angustifolia Roxb. of Rubiaceae -shrubby plant in the margin of temporary water body 


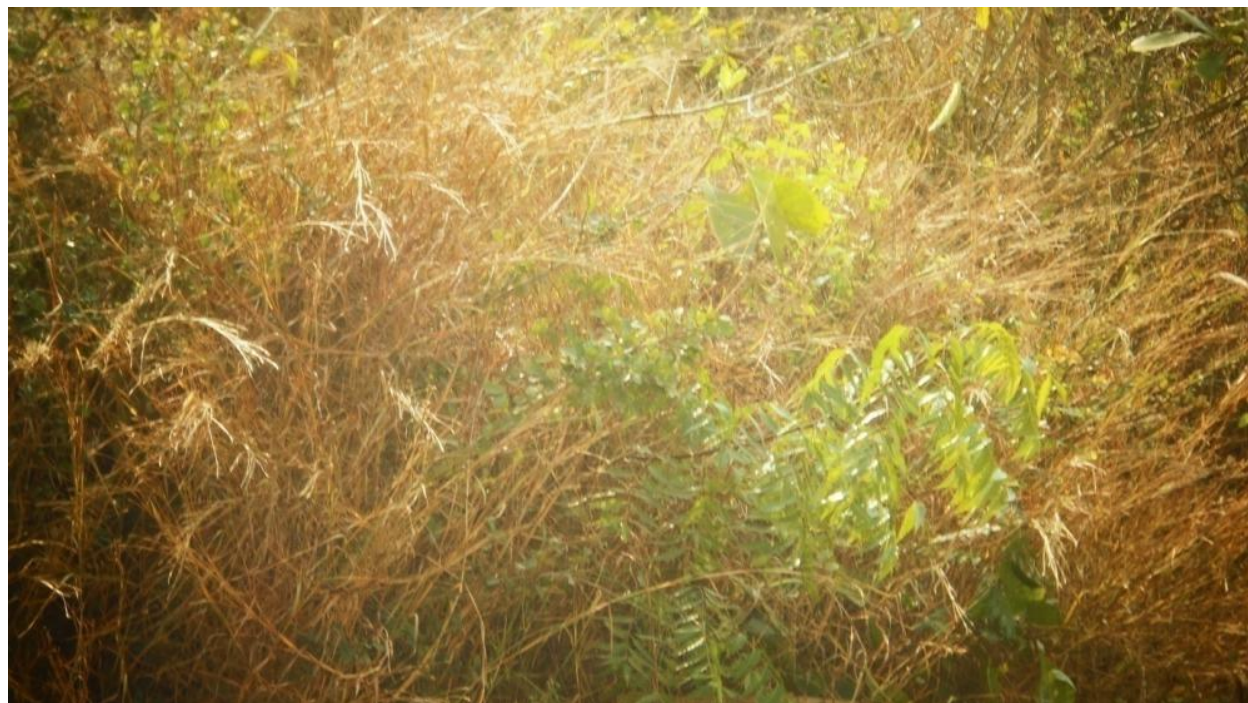

Fig. 29 Neem Tree showing compound leaves (Azadirachta indica A. Juss. )- important medicinal plant.

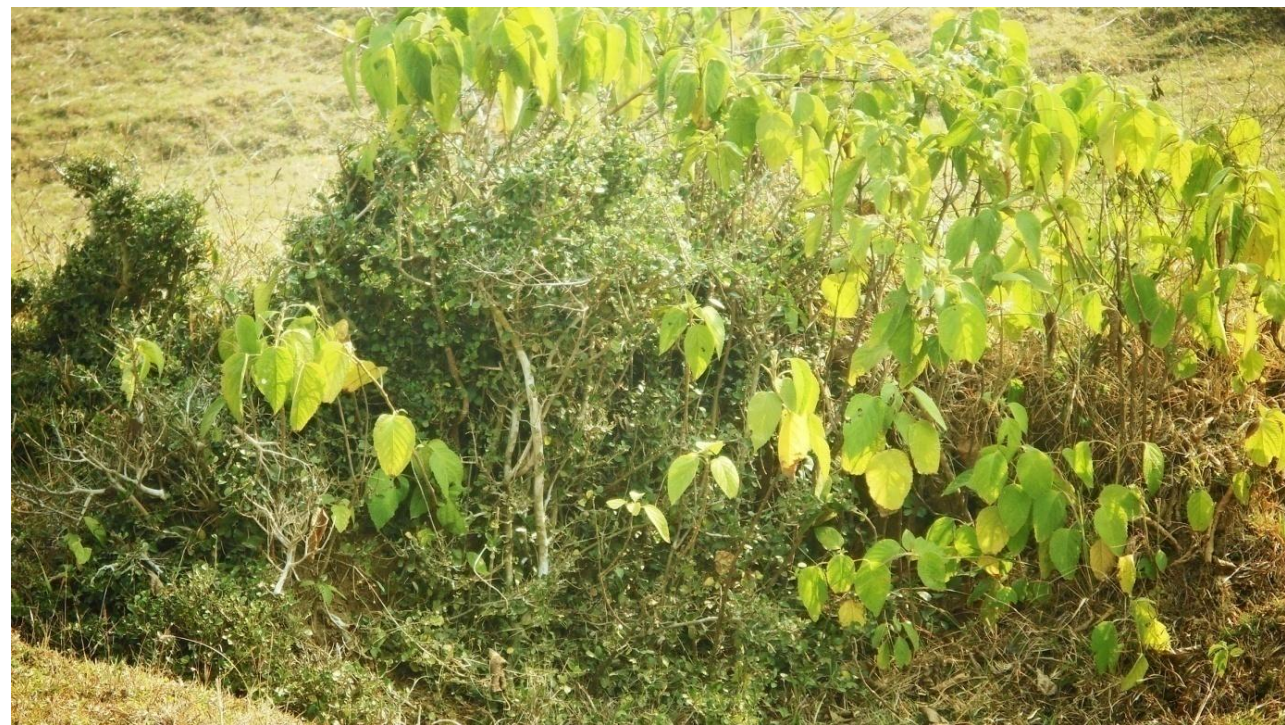

Fig. 30 Dark green dwarf plant at the left is Streblus asper Lour. and yellow green broad leaved plants are Clerodendrum viscosum Vent. (Beng.-Seora and Ghetu association)

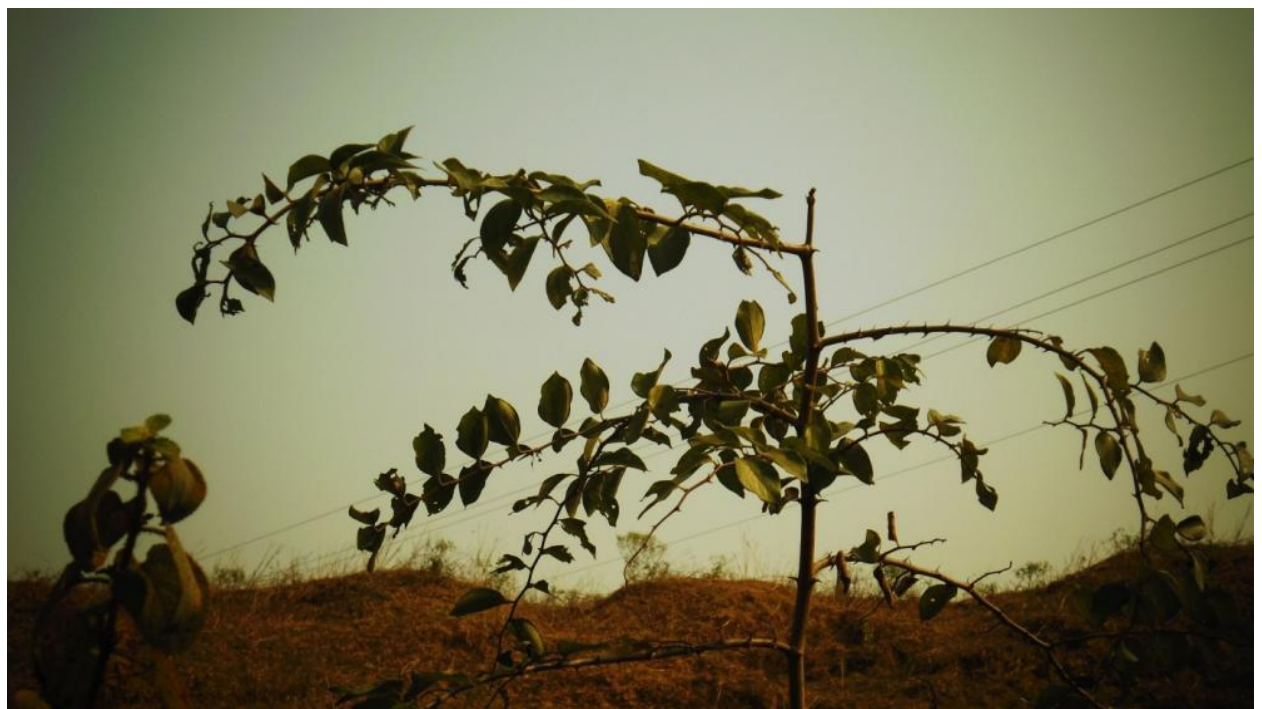

Fig. 31 Ziziphus oenoplea (L.) Mill. of Rhamnaceae-a plant used to prepare living fence 


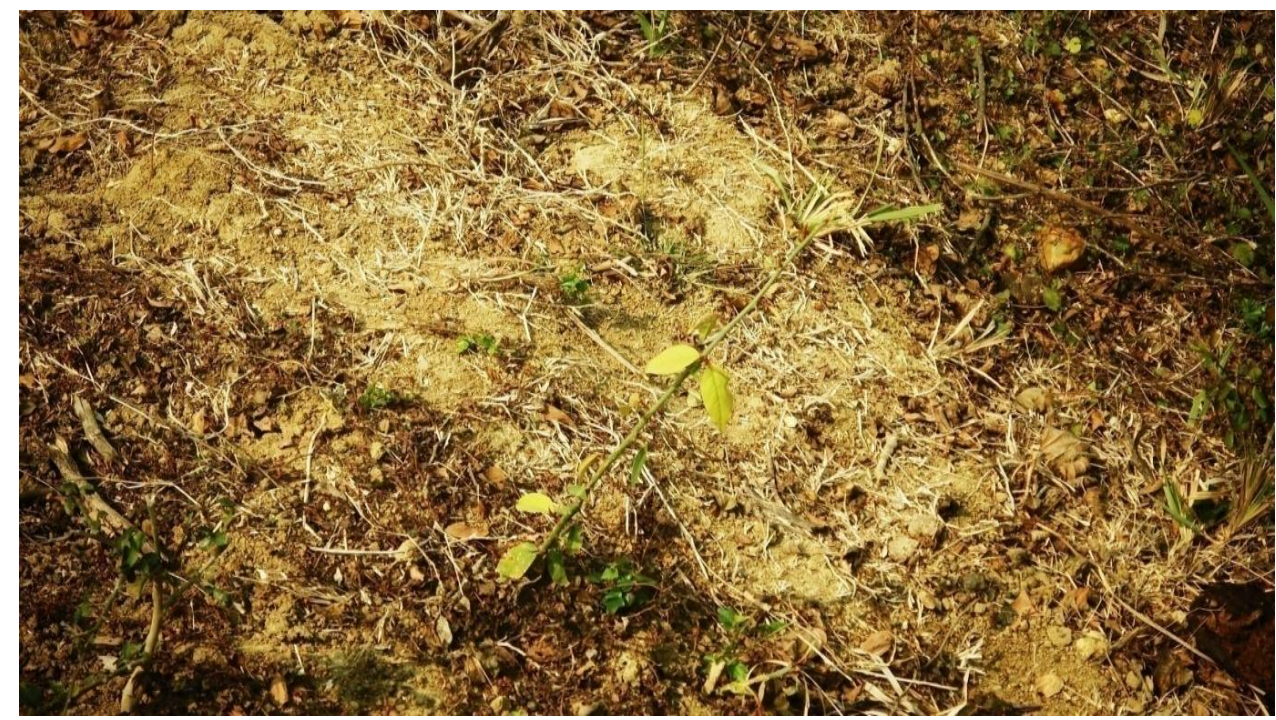

Fig. 32 Capparis sepiaria L. of Rhamnaceae is a medicinal plant

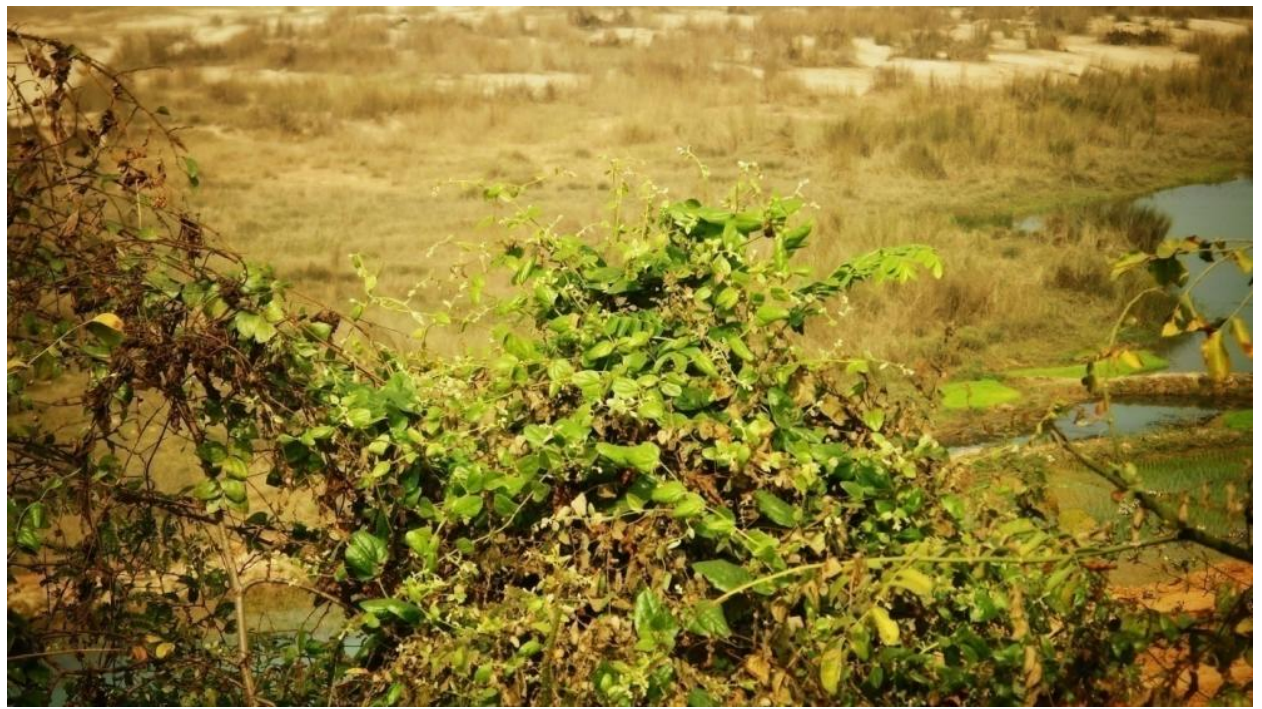

Fig. 33 Cocculus hirsutus (L.) Diels of Menispermaceae-used to prepare green curd from leaf decoction

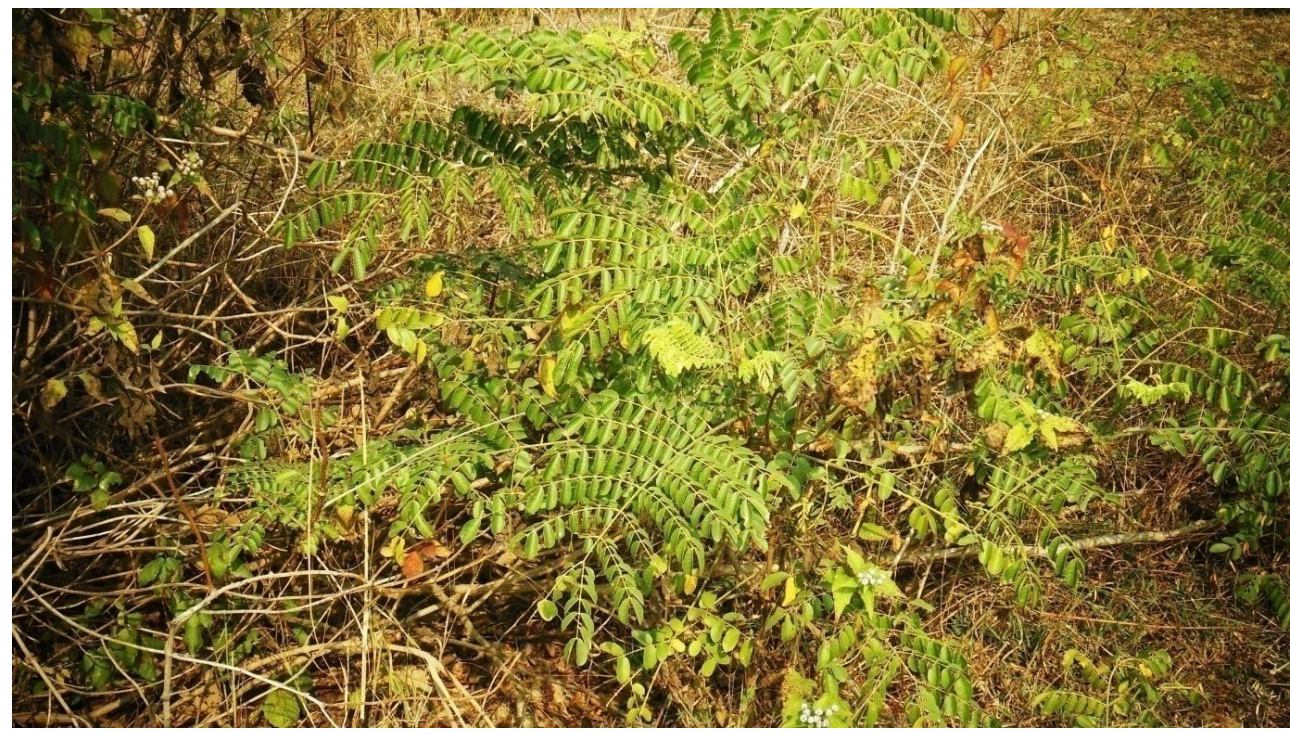

Fig. 34 Plant with compound leaf is Caesalpinia bonducella (L.) Fleming of Caesalpiniaceae-an anthelmintic plant, roasted kernel of seeds used widely. 


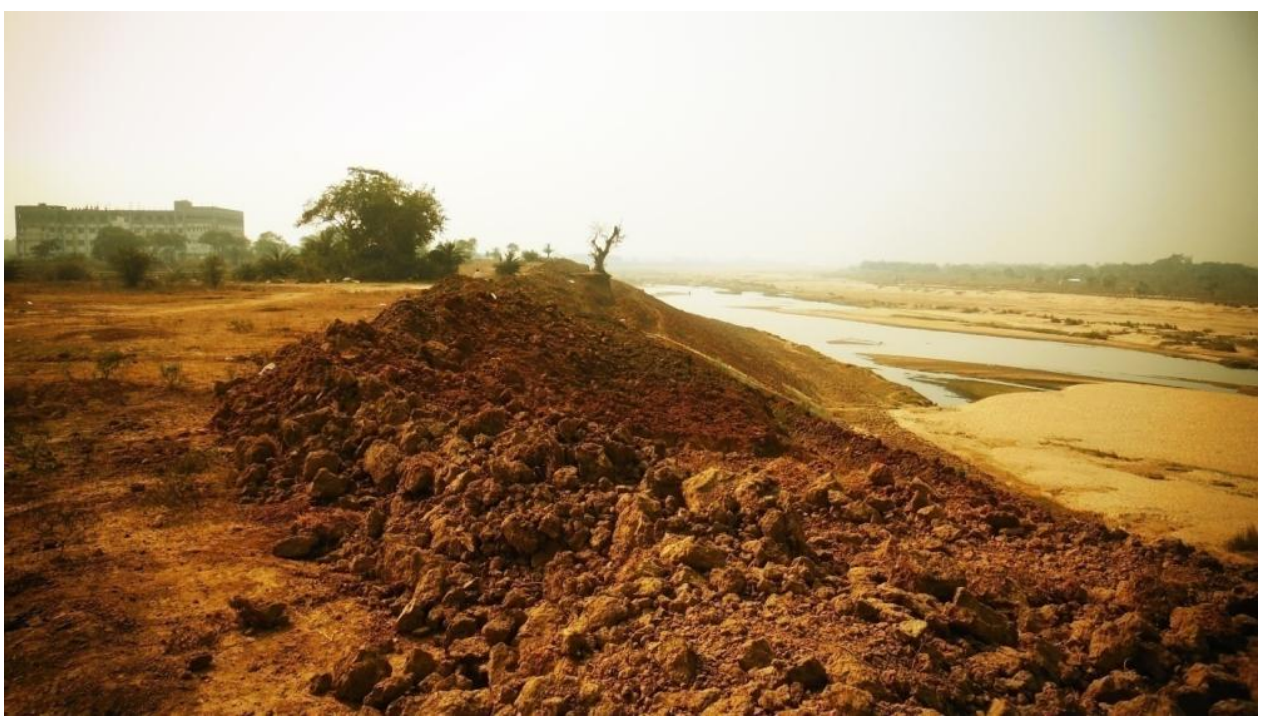

Fig. 35 Degraded site of Kansai and backside of the river bank showing Lalgarh Government College

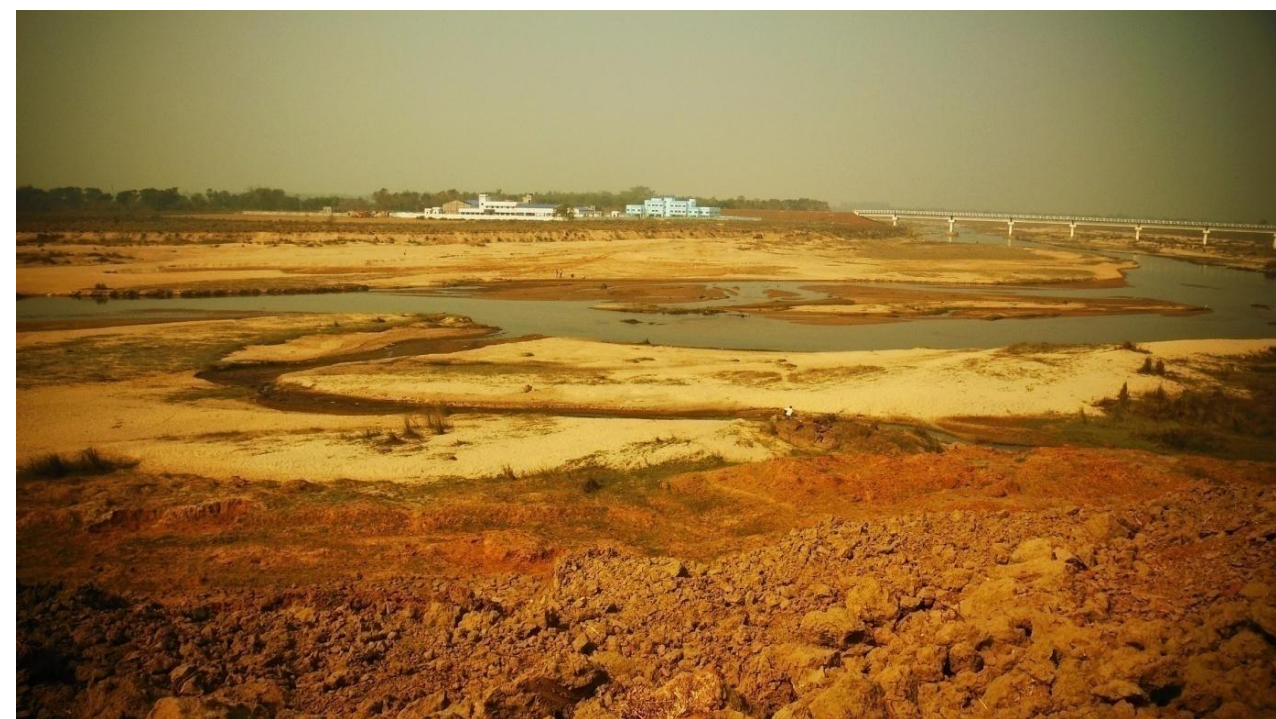

Fig. 36 Landscape showing Amkala Bridge and educational institute-a neglected site now going to flourish

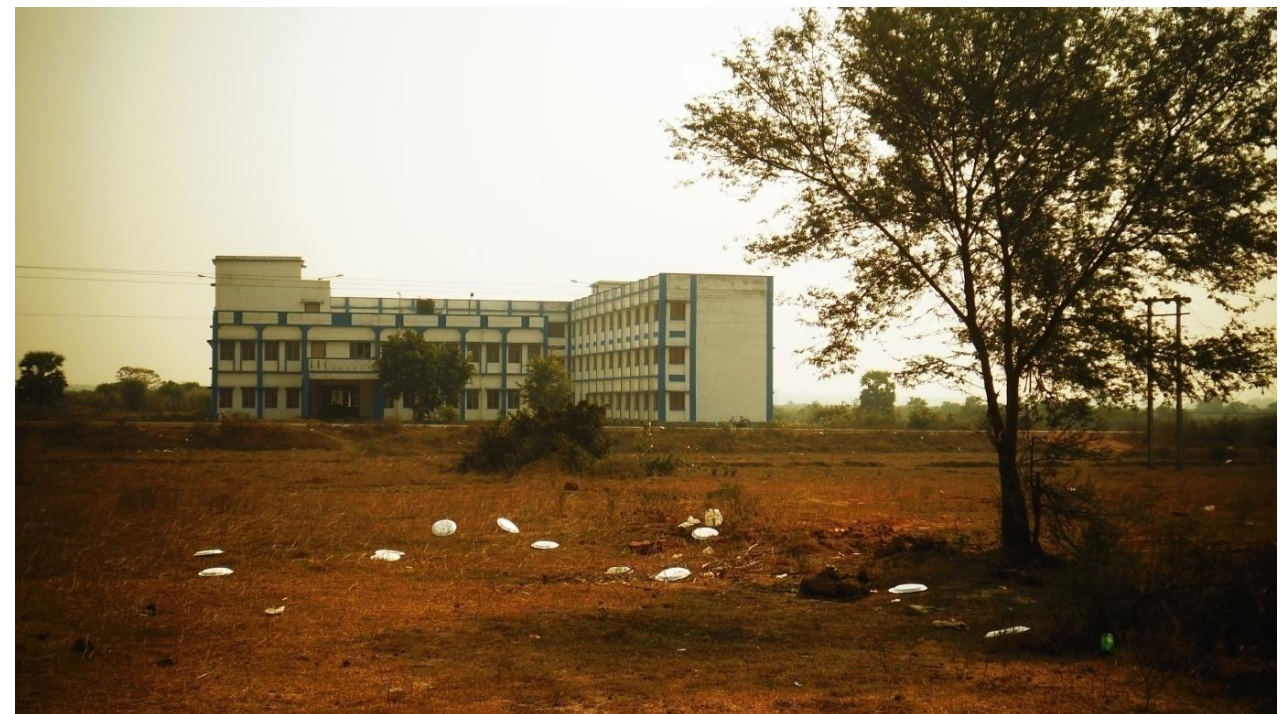

Fig. 37 Newly built model School opposite to which exhibit picnic spot showing plastic pollution need awareness to protect environment 


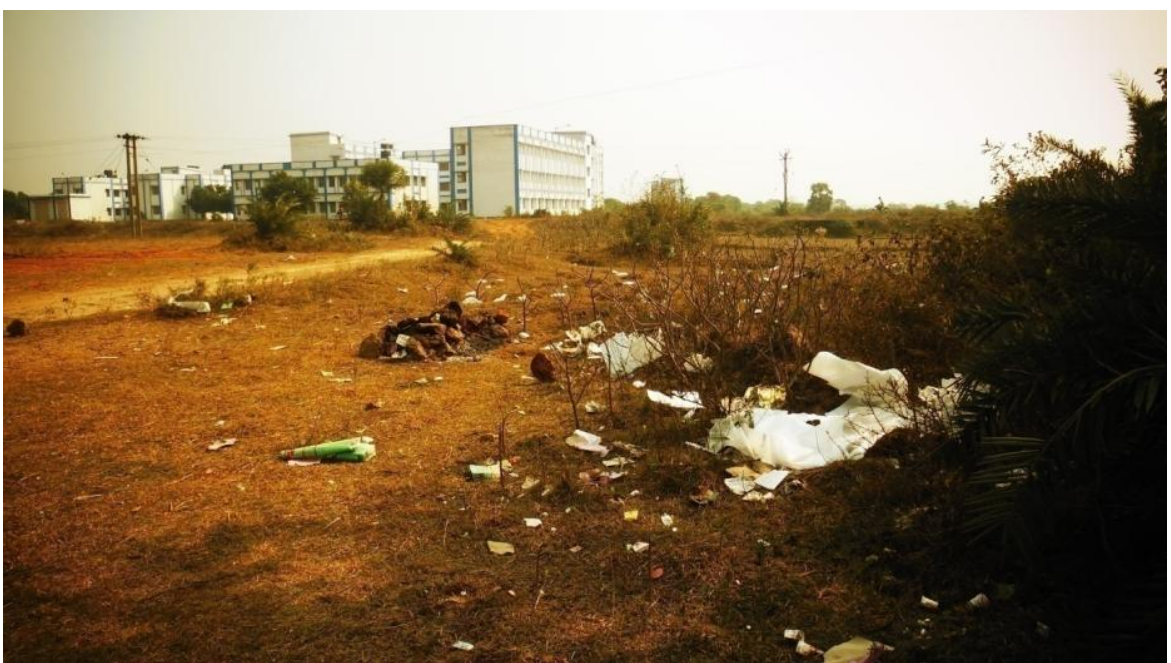

Fig. 38 Seriously damaged site under threat due to plastic pollution lead to vision pollution also

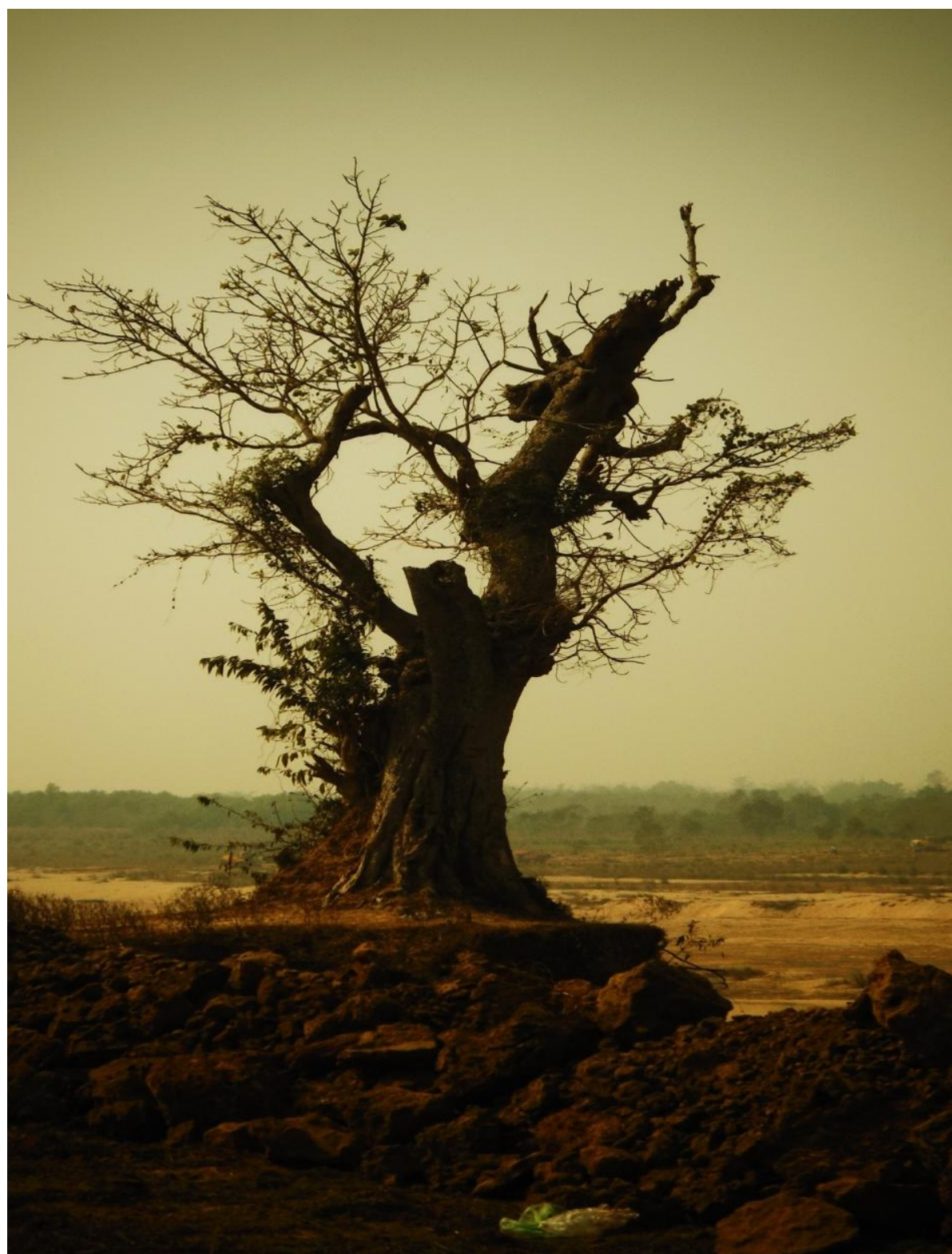

Fig. 39 Old Banyan tree (Ficus benghalensis L. tree) now going to vanish from the site as the bank is fragile 


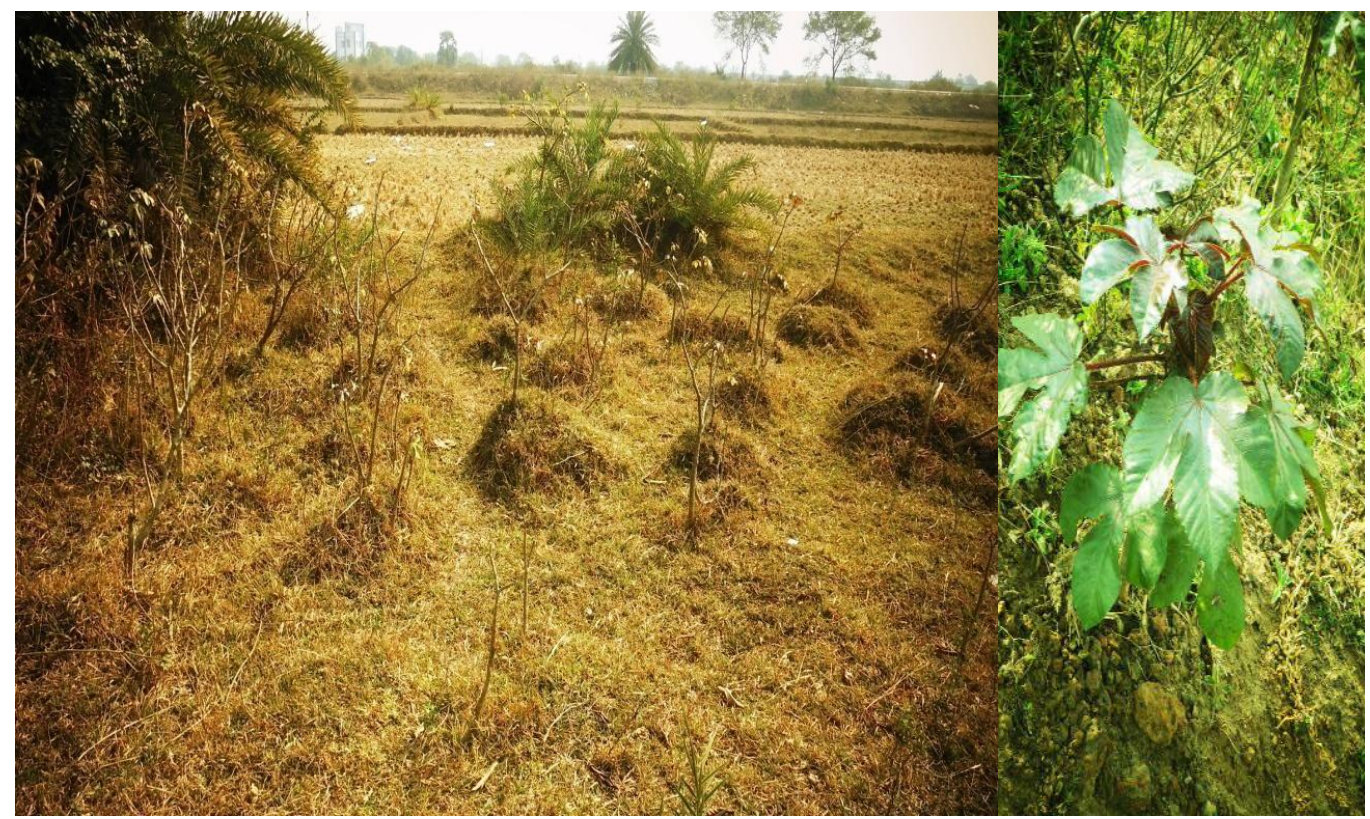

Fig. 40 Jatropha gossypifolia L. plants of Euphorbiaceae in front of date palm, right one whole plant

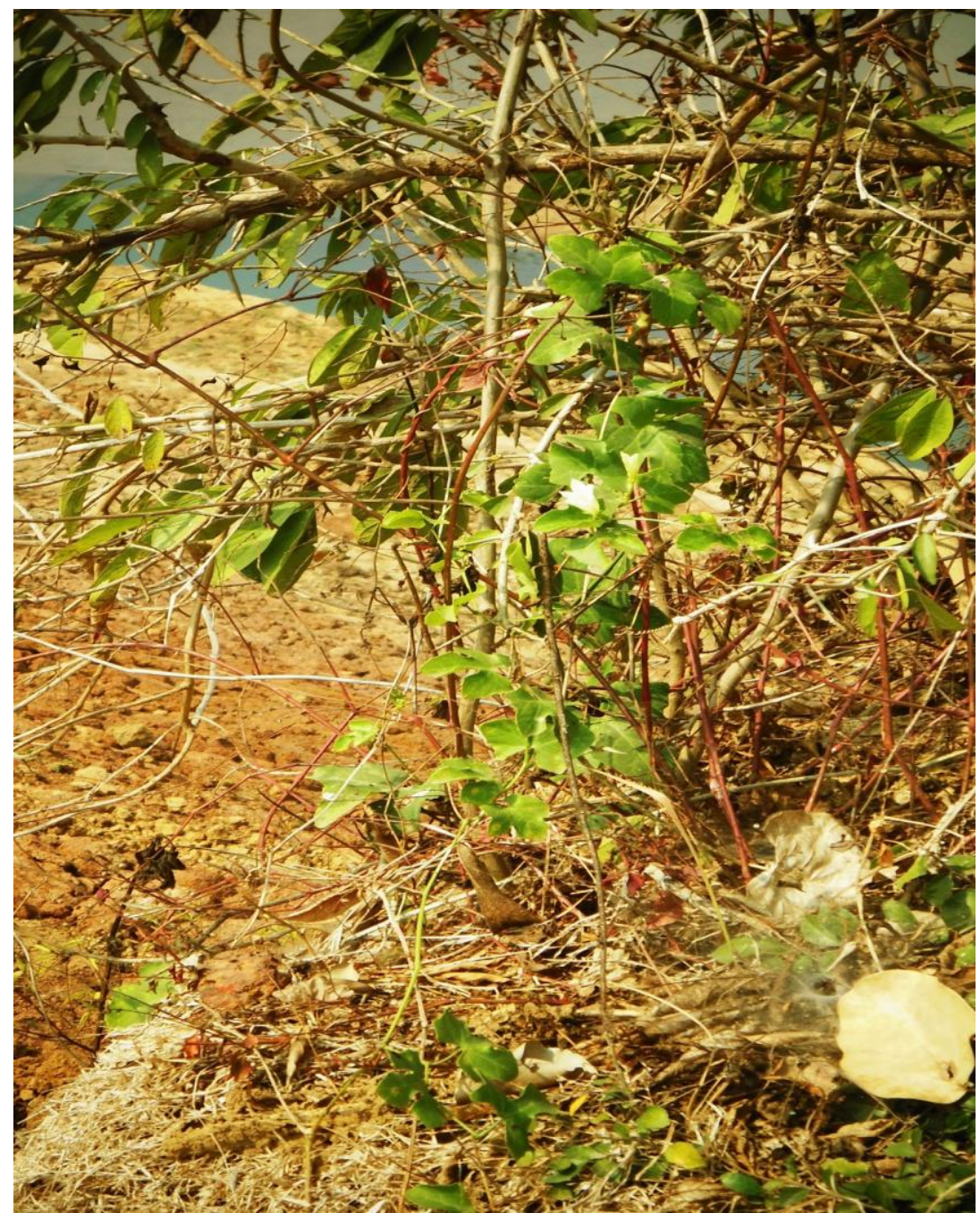

Fig. 41 Climber with white flower is a plant Coccinia grandis (L.) Voigt. of Cucurbitaceae-medicinal plant used in boils 


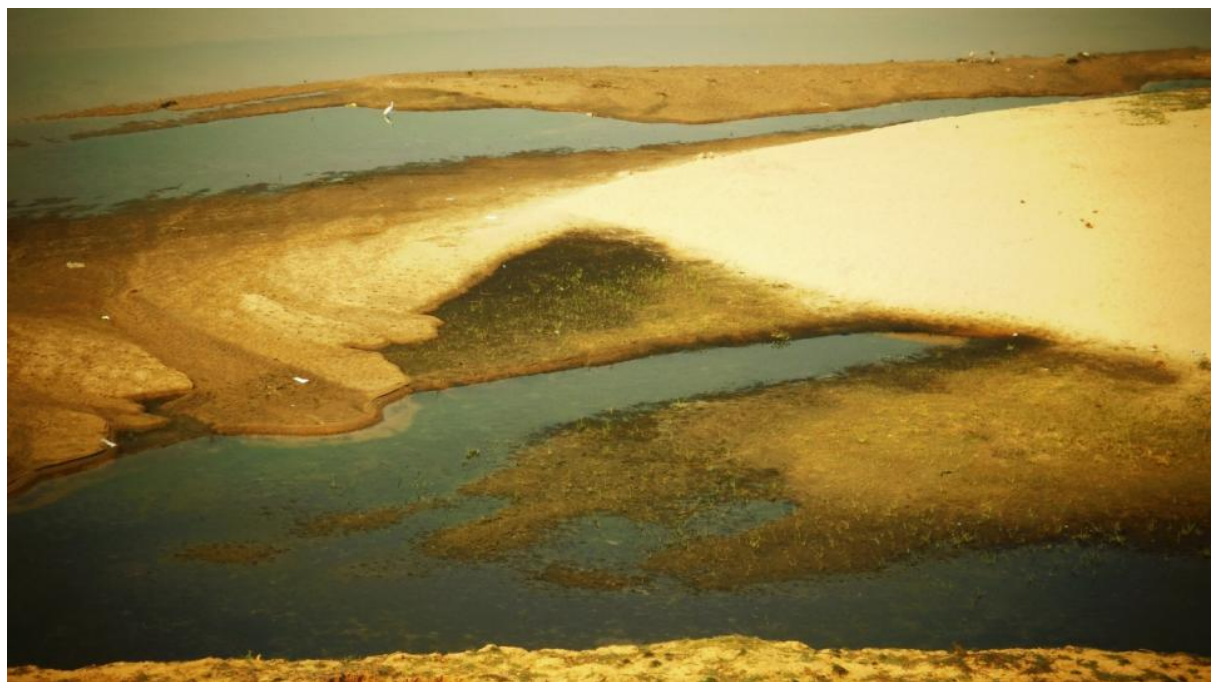

Fig. 42 Dark green patches in river Kansai at Lalgarh showing mingled threads of algae dominated by Spirogyra spp.

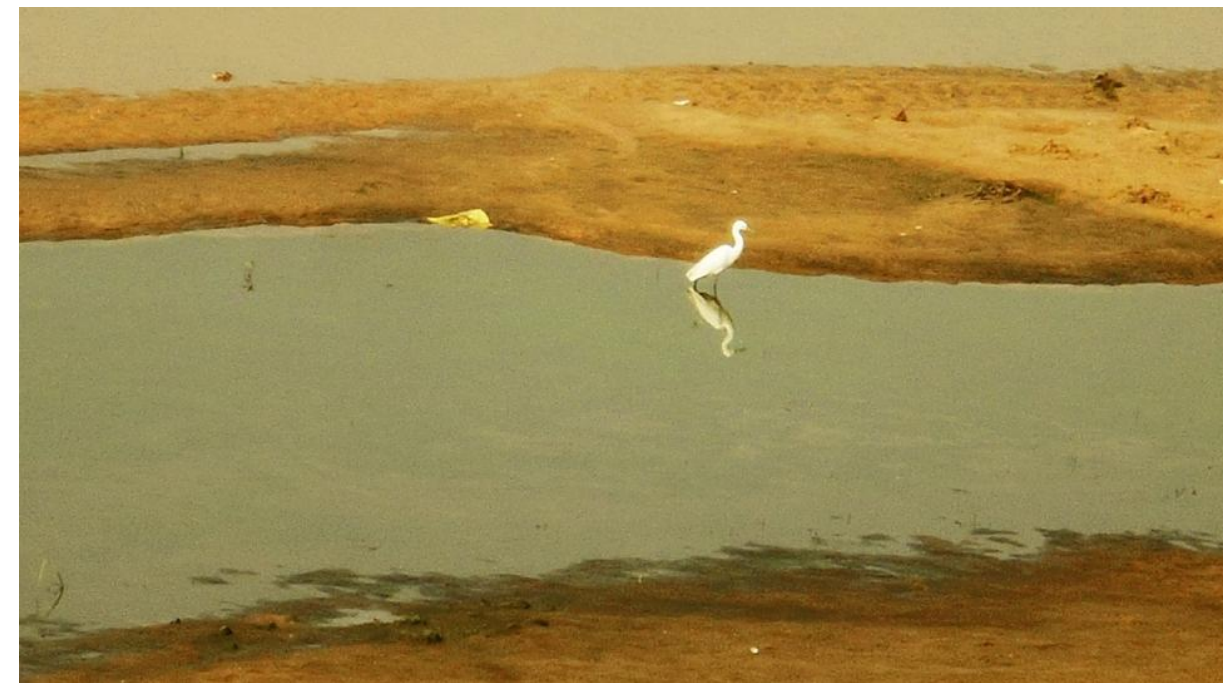

Fig. 43 Degraded river belt showing least birds due to lesser availability of fish and zoo plankton, note that the white bird is Egret

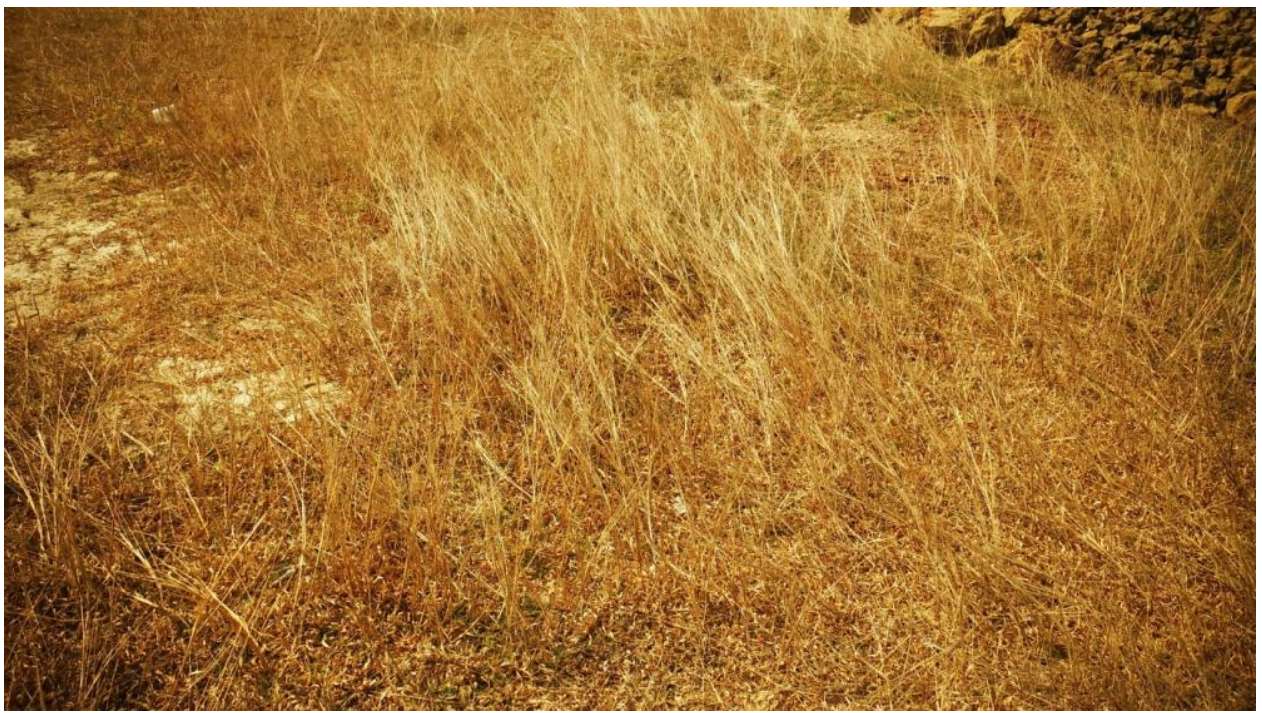

Fig. 44 Dry Love grass i.e. Chrysopogon aciculatus (Retz.) Trin. of Poaceae during winter though the underground rhizome is still alive 


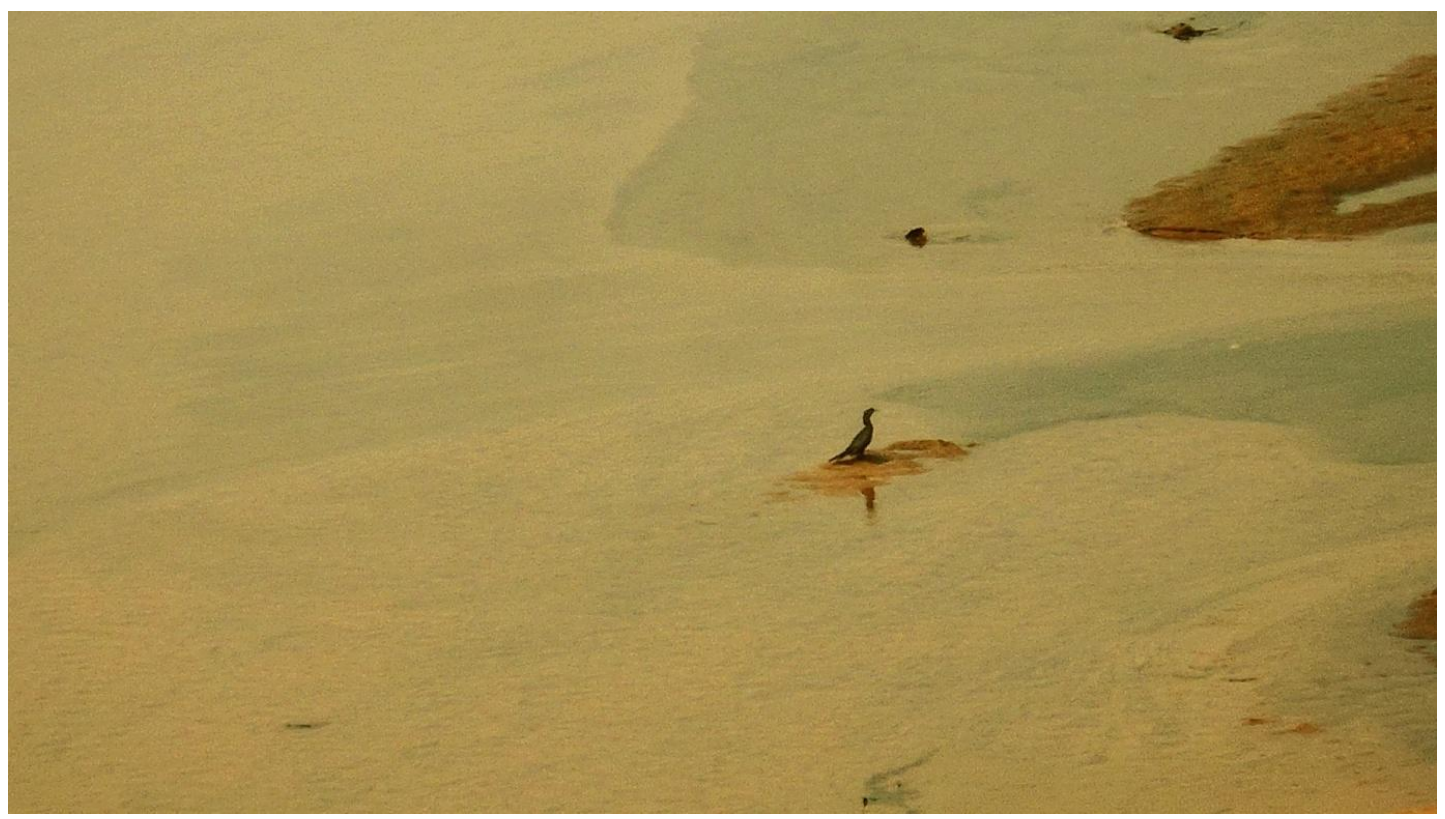

Fig. 45 Lone Little Cormorant in river basin of Kansai at Lalgarh

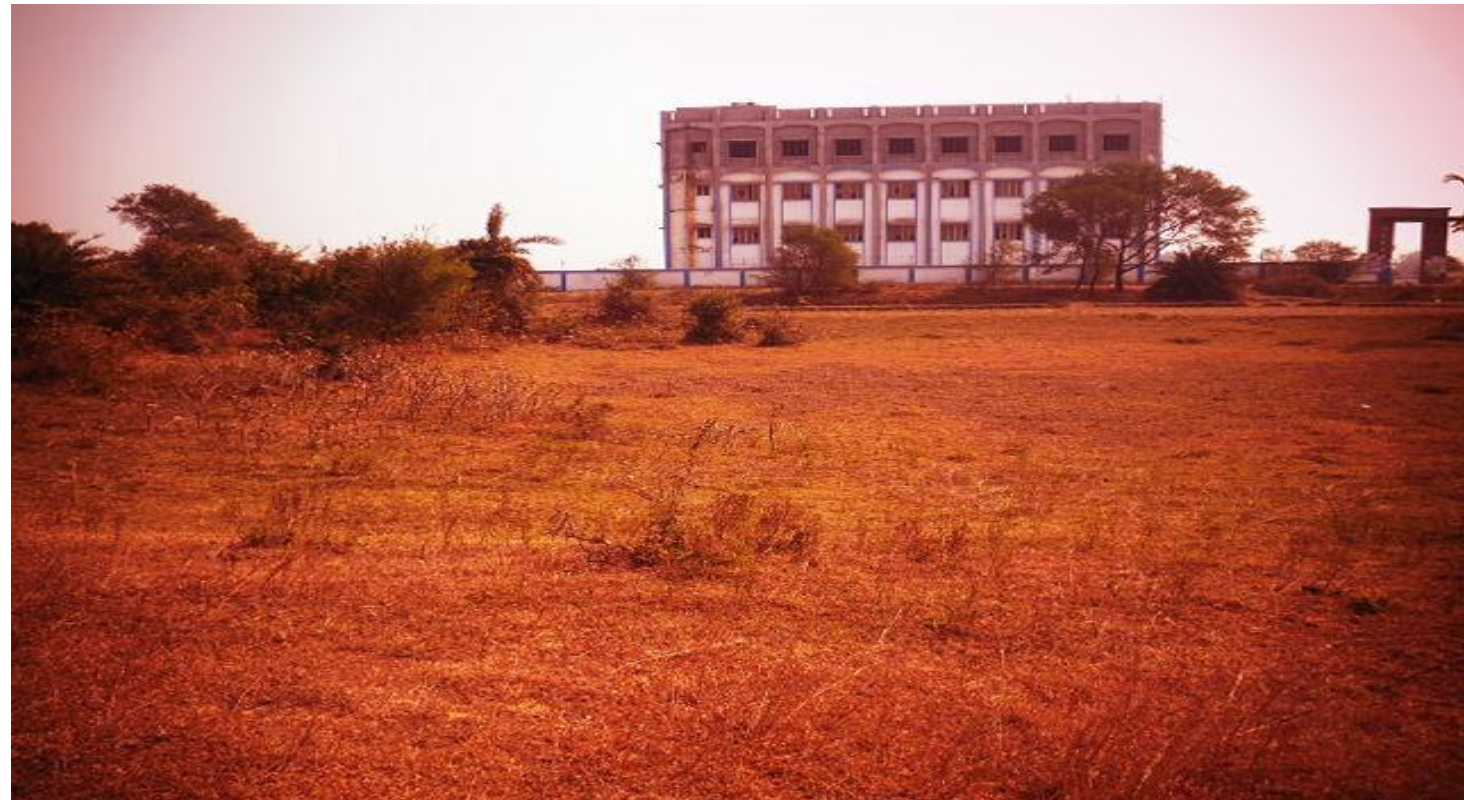

Fig. 46 Degraded land used as grazing field in front of Lalgarh Government College at Lagarh, note that huge Acacia trees growing here and there which is used as fodder tree

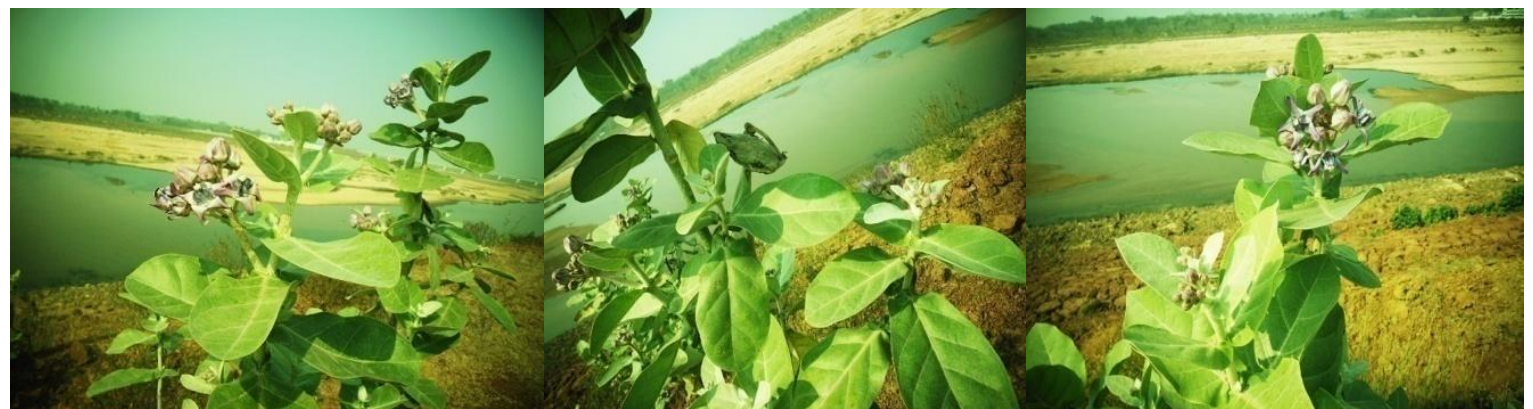

Fig. 47 (a-c) Calotropis gigantea (L.) Ait. of Asclepiadaceae is nearer to river Bank- a medicinal plant and a fodder plant 
Flora of Kansai Basin at Lalgarh of Paschim Medinipur District in West Bengal with Special ..

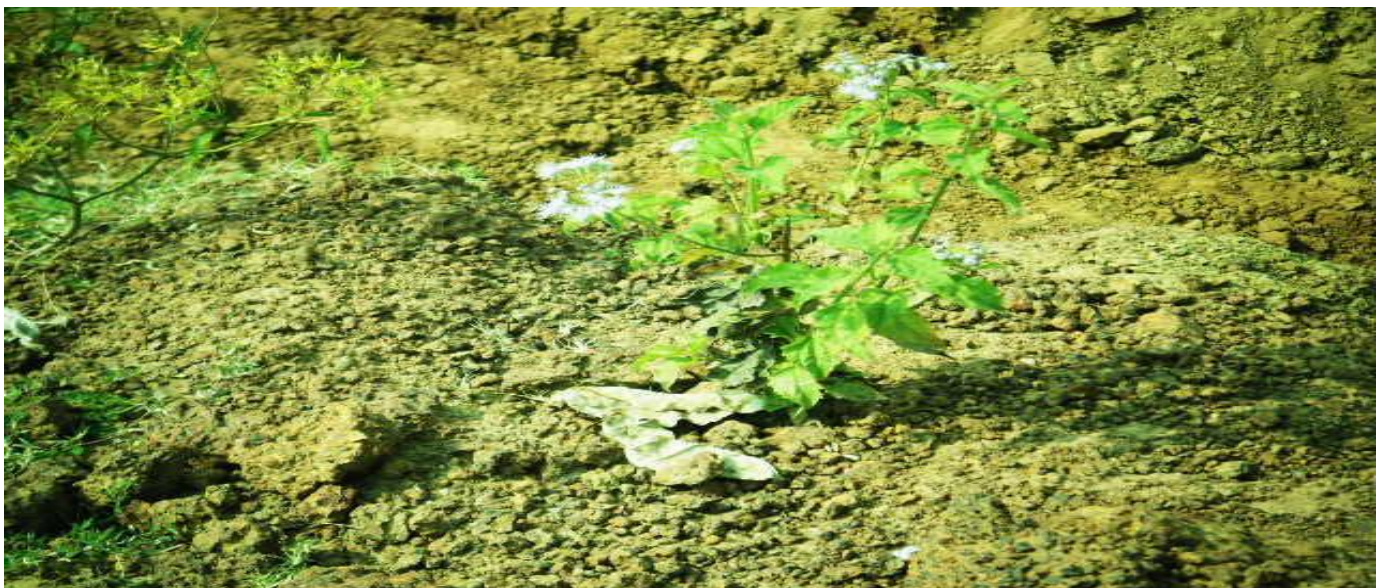

Fig. 48 Eupatorium odoratum L. of Asteraceae and exotic species established everywhere as exotic species

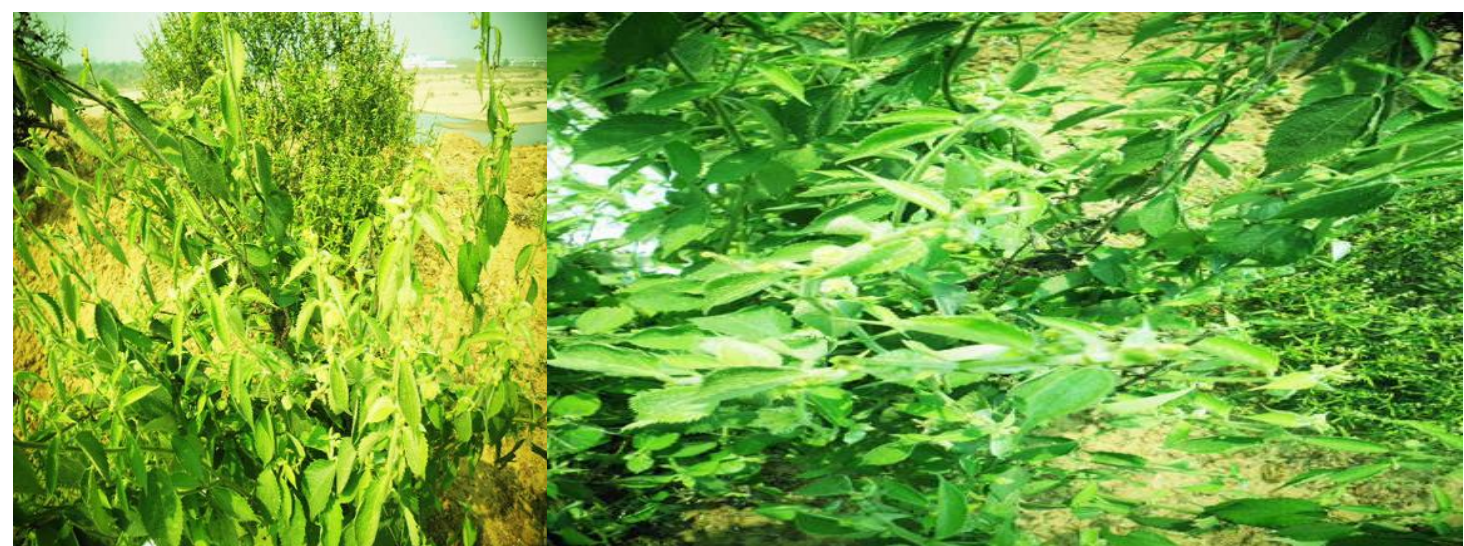

Fig. 49 (a-b) Tragia hispida Willd. of Euphorbiaceae is a medicinal plant

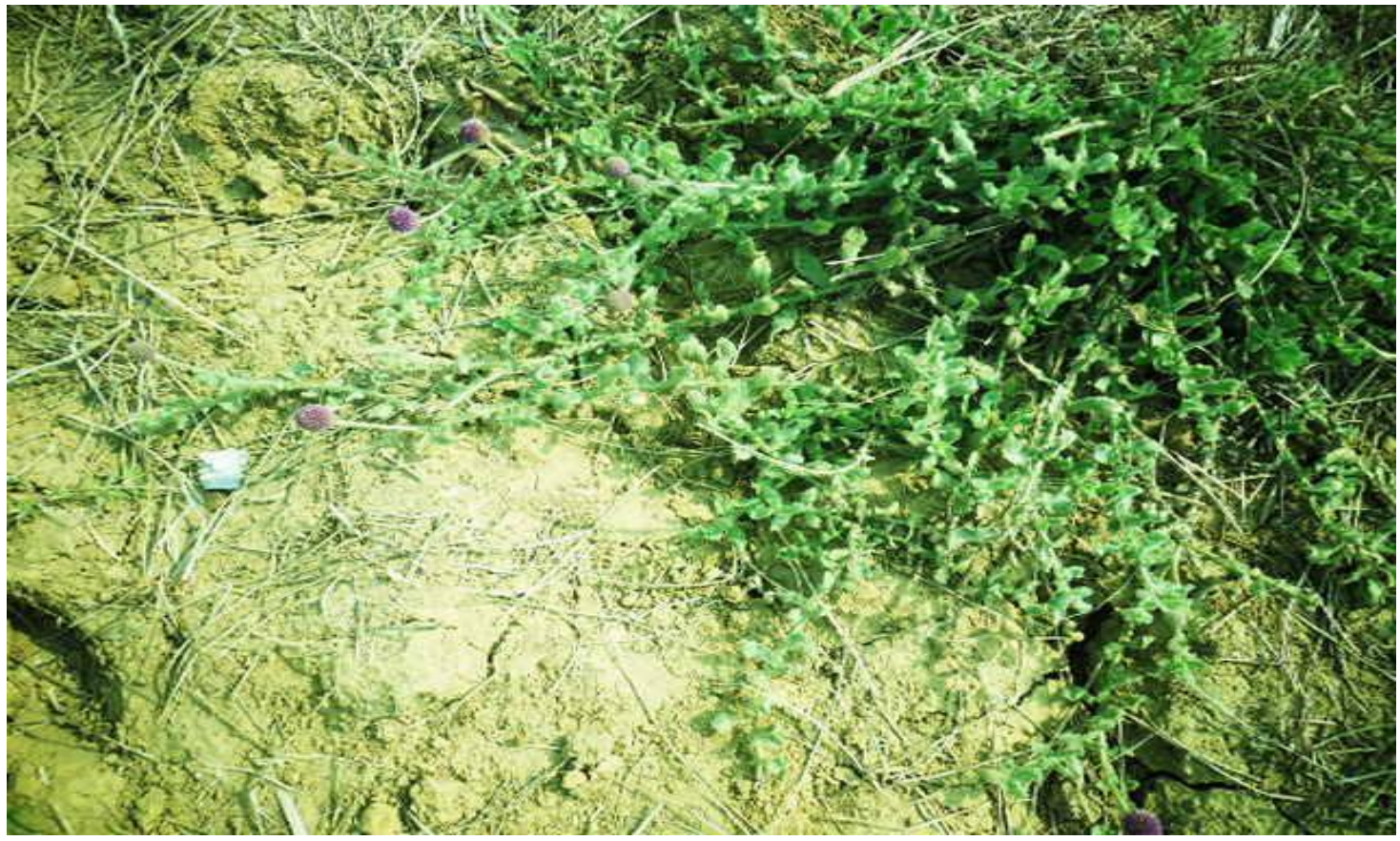

Fig. 50 Spharenthus indicus L. of Asteraceae 


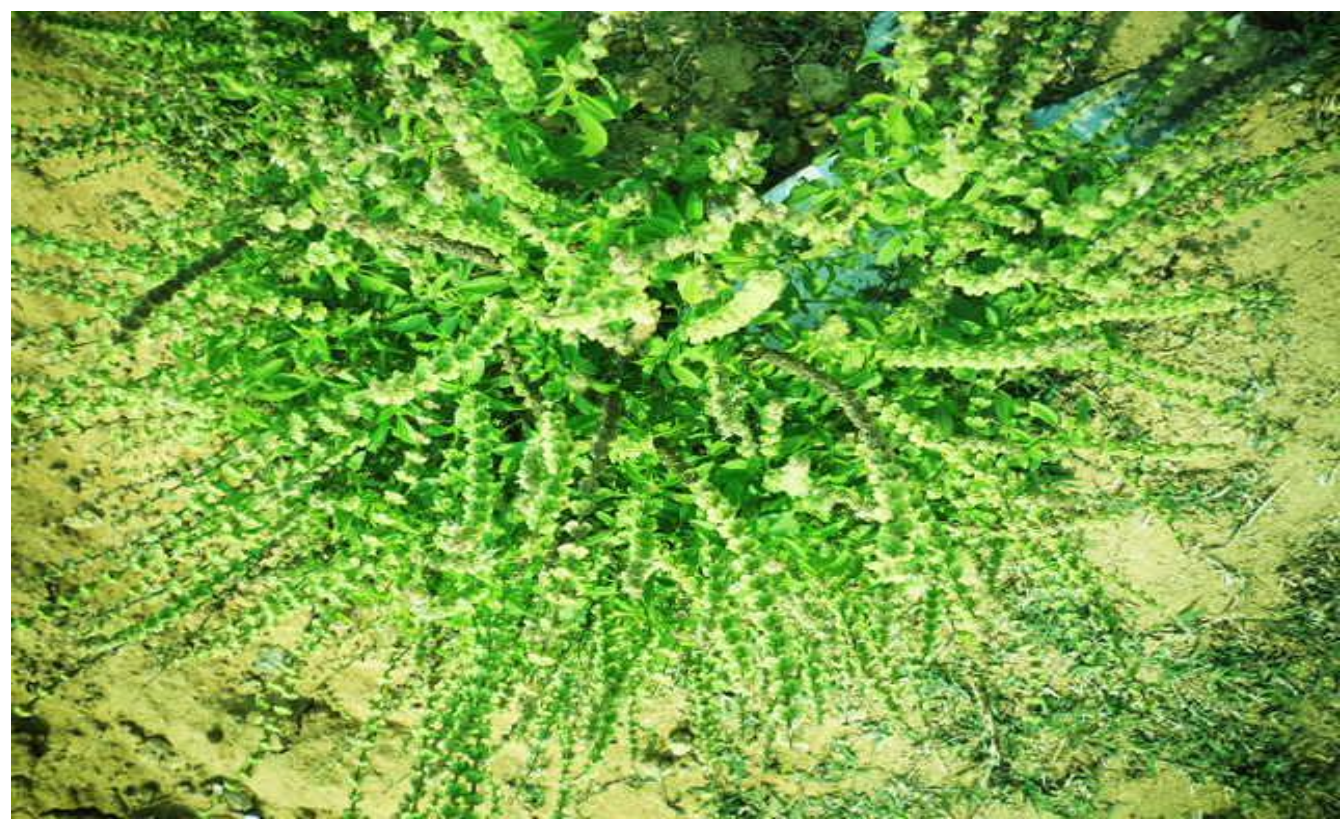

Fig. 51 Ocimum americanum L. of Lamiaceae (Labiatae)- An important medicinal plant of waste land

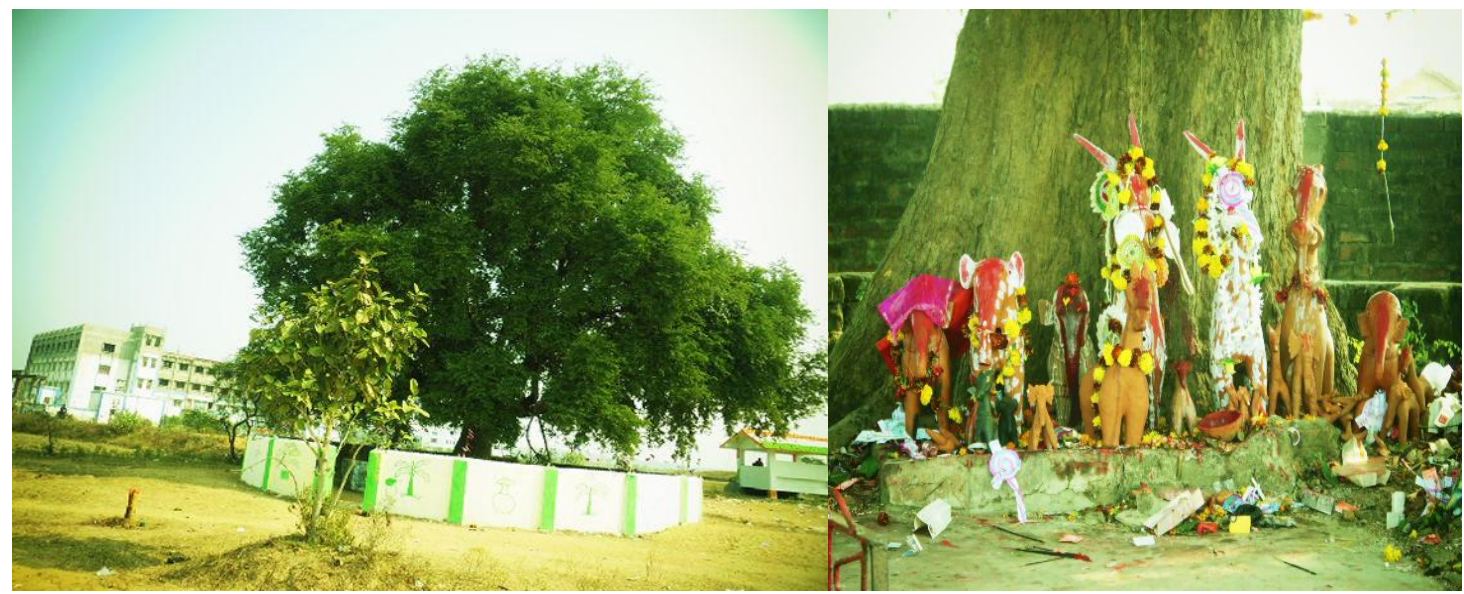

Fig. 52 Sankhasini Sacred grove conserving Tamarind tree, right one 'idols' of Sacred grove beneath the tree (Tamartindus indica L. of Caesalpiniaceae)

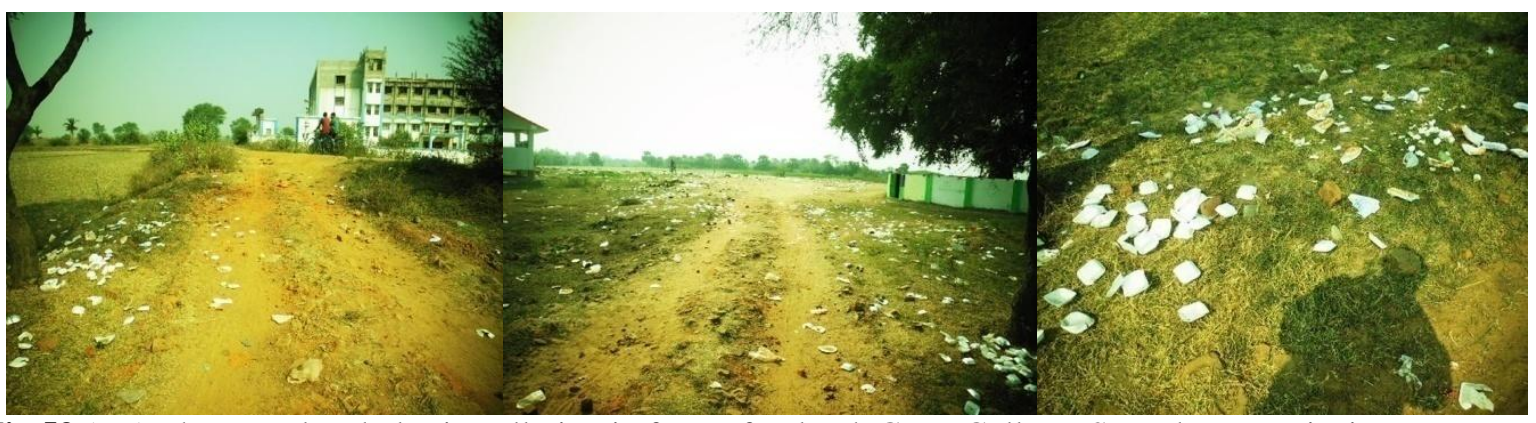

Fig 53 (a-c) Thermocol and plastic pollution in front of Lalgarh Govt. College, Scared grove, picnic spot

\section{Conclusion}

The study is important because this is the first time report on the bank community of Kansai at Lalgarh which could be the starting point of taxonomic as well as ecological research. The record may be a general Key guide to the beginners and amateur field worker in the field of Botany, Zoology, Environmental Study and nature lovers to use the data for future study. The land planner and policy maker may be made the new plan to protect the habitat as the habitat is facing threat. Pollution and human pressure causing the site more and more fragile which need immediate protection to protect the site threat less or more pristine. 


\section{Acknowledgements}

I acknowledge authority of my parent organization i.e. Higher Education Directorate, Govt. Of West Bengal, India for giving me transfer from Darjeeling Govt. College, Darjeeling to Lalgarh Govt. College, Lalgarh, Paschim Medinipur for teaching and extension including research. I thank my students of Botany Deptt. for help as and when required. Local people who helped me in field are well acknowledged.

\section{Further Readings}

[1]. Ambasht, R. S., Singh, M. P. and Sharma, E. An environment study of soil and water Conservation through herbaceous plants. National Academy of Science Letters, 1984, 6 (5): 143.

[2]. Anonymous Flora of West Bengal, Vol.-I, BSI, Kolkata, Flora of India, Series-2, 1997.

[3]. Anonymous Medicinal Plant Resources of South West Bengal, Vol.-I, Research Wing, Directorate of Forests, Govt. of West Bengal, 2005.

[4]. Anonymous Medicinal Plant Resources of South West Bengal, Vol.-II, Research Wing, Directorate of Forests, Govt. of West Bengal, 2010.

[5]. Aandyopadhyay, K. B.. Amader Rajya (Bengali Version), Kishore Gyan Vigyan Prakashani, Spectrum Offset, Kolkata-37, 2009, pp. 76.

[6]. Banerjee, L.K., Rao T.A., Sastry, A.R.K. and Ghosh, D. Diversity of the Coastal Plant communities in India. ENVIS \& EMCBTAB. Botanical Survey of India, BSI: Kolkata. 2002.

[7]. Basak R. K. and Guha Bakshi, D. N. Floristic studies on the state of West Bengal: Present status and future strategies. Bull. Bot. Surv. India. 1977, 19 (14):42-48.

[8]. Beddel, P.E. Seed Science and Technology, Indian Forestry Species, Allied Publishers Limited, New Delhi, 1998, pp.1-346.

[9]. Bentham, G and Hooker, J. D. Genera Plantarum. London: Reeve \& Co.1-3. 1862-1883.

[10]. Bestelmeyer, B.T., Trujillo, D.A., Tugel, A. J., and Havstad, K.M. A Multi-Scale classification of Vegetation dynamics in arid lands: What is the right scale for models, monitoring, and restoration?, Journal of Arid Environments, 2006, 65: 296-318.

[11]. Bilgrami, K.S. The Living Ganga, Narendra Publishing House, Delhi., 1919. Chakrabarty, S. Coastal Environment of Midnapore, West Bengal: Potential Threats and Management, Journal of Coastal Environment, 2010; I: 27-40.

[12]. Das, A. A. and Das, D. Preliminary Studies on Common Birds of West Bengal with Special Reference to Vegetation Spectrum, India , IOSR-JESTFT, 2016, 10 (11): 12-34.

[13]. Das, D Community study of plants species in coastal areas of Mohana and old Digha of Purba Medinipur District with special reference to Eco-sustenance, Indian Journal of Applied and Pure Biology. 2014, 29(2): 255-266.

[14]. Das, D Community study of plant species in coastal areas of Mohana and Old Digha of Purba Medinipur District with special reference to Eco-sustenance of life in near future, India J. Applied \& Pure Bio., 2014, 29 (2): 255-266.

[15]. Das, D. Study of Vegetation Ecology of Forests of South West Bengal with special reference to Non-Timber Forest Produce (NTFPs) Productivity, Ph. D Thesis awarded from Vidyasagar University, West Bengal, 2007, (Work From CNH, Botanical Survey of India, Shibpore, Howrah, West Bengal).

[16]. Das, D. Ecological status of plants in sacred groves of southwest Bengal (Midnapore, Bankura and Purulia District) of West Bengal, Final UGC-Project Report, 2009, PSW-160/06-07(ERO) dated 19.02.2007, 2009.

[17]. Das, D. Final Project Report on 'Ecological studies of Vegetation in coastal areas of Purba Medinipur under stress for sustenance of life', UGC-Project report (No. PSW-087/11-12 (ERO), Kolkata, dated 23.04.2013., 2015.

[18]. Das, D. Ecological Studies on Jhitka Forest Under Medinipur Forest Division, IJSART, 2016, 2 (12): $296-302$.

[19]. Das, D and Das. M. Vegetation Ecology of Coastal belt of Khejuri area of Purba Medinipur District with special reference to Hijli Coast, West Bengal, India, IOSR-Jour of Pharmacy, 2014, 4 (2): 2319-4219.

[20]. Das, D and Ghosh, P Ecological Studies of Ecosystem Health Indicators at Nayagram of Paschim Medinipur District in Lateritic forests of Southwest Bengal, India, IOSR Journal of Environmental Science, Toxicology and Food Technology, 2014, 8 (6): 48-63

[21]. Das, D. Eco-tourism and Eco-degradation in Darjeeling Himalaya, West Bengal, Abstract and Full Length Paper in a seminarVariation and prospects of Eco-Tourism at Darjeeling and Dooars, 20 ${ }^{\text {th }}$ December, 2016, funded by Higher Education Department, Govt. of W.B., at Gorubathan Govt. College, Darjeeling, India.

[22]. Dash, M.C. and Das, S.P. Fundamentals of Ecology, Third Edition, The McGrew-Hill Companies, Tata McGrew-Hill Education Private Limited, New Delhi., 2010, pp.1-562.

[23]. De, D. K. Grass Flora of Medinipur District. Ph.D. Thesis, Vidyasagar University, West Bengal. 2002.

[24]. Ganguli, S., Gupta, H. and Bhattacharya, K. Vegetation structure and species diversity in Garhjungle sacred forest, West Bengal, International Journal of Environmental \& Agriculture Research, 2016, 2 (9): 72-79.

[25]. Gripson, S. Greipsson, S. Restoration Ecology, Jones \& Bartlett Learning, USA, 2011, pp-387.

[26]. Groom, M.J., Mmeffe, G.K., Vcarroll, C.R. and contributors Principles of Conservation Biology, Third Edition, Sinauer Associates, Inc. Publishers, USA, 2006, pp.-793.

[27]. Haines, H. H. The Botany of Bihar and Orissa, Vol. I-IV, BSI, Calcutta, 1921-1925.

[28]. Holland, M. M., Risser, P. G. and Naiman, R. J. Ecotones: The role of land scape boundaries in the management and restoration of changing environments, Chapman \& Hall., New Delhi, 1991.

[29]. Hooker, J. D. Hooker, J. D. Flora of British India, Vol. 1-VII, 1892-1897, BSI, Calcutta.

[30]. Jorgensen, S.E., Xu, fu-liu and Costanza, R. Hand Book of Ecological Indicators for Assessment of Ecosystem Health, Second Edition, CRC Press, New-York, 2010, pp.-484.

[31]. Maji , S. and Sikdar, J.K.Sedges and grasses of Midnapore District, West Bengal, J. Econ Taxon Bot. 1984, 4(1): 233-254.

[32]. Mishra, R. Ecology Work Book, Oxford and IBH Publishing Company, New Delhi, 1968.

[33]. Mitsch, W.J. and Gosselink, J.G. The role of riparian corridors in maintaining regional bio-diversity, Ecol. Appl. 1993, 3: 209-212 
[34]. Mukherjee, A.K., and Banerjee, L. K. Three new plant records along Midnapore coast of West Bengal, J Bombay Nat Hist Soc. 1968, 65:268-269.

[35]. Mukherjee, A.K. and Chaterjee, S. Coastal erosion and accretion at and around Digha in Midnapore District of West Bengal. Indian Journal Geography and Environment. 1997, 2: 1-11.

[36]. Mukherjee , A.K. Mangrove Wealth of Indian Sundarbans - Utilisation and conservation. J Econ Taxon Bot. 1984, 5(1): 227-230.

[37]. Popradit, A., Srisatit, T., Kiratiprayoon, S., Yoshimura, J., Ishida, A., Shiyomi, M., Murayama, T., Chantaranothai, P., Outtaranakorm, S; and Phromma, I. Anthropogenic effects on a tropical forest according to the distance from human settlements, Scientific Reports, 5-14689:2015, pp. 1-10 doi.: 10.1038/srep14689

[38]. Prain, D. Prain, D. Bengal Plants, Vol.-I-II, (Revised Edn.), BSI, Calcutta, 1963.

[39]. Rao, T.A., Mukherjee, A.K., Banerjee, L. K. Vascular plants of the coastal Midnapur district, West Bengal. Indian For. 1970,96 : $668-677$.

[40]. Rao, R. R. and Sharma, B. D. Rao, R. R and Sharma, B. D. A Manual for Herbarium Collections, BSI, Brabourne Road, 1990, Kolkata-1.

[41]. Raunkiaer, C. The-life forms of plants and statistical plant Geography, Oxford University Press, Oxford, 1934.

[42]. Gottschling, M., Miller, J. S., Weigened, M and Hilger, H.H. Congruence of a phylogeny of Cordiaceae (Boraginaceae) inferred from ITS sequence Data with morphology, Ecology and Biogeography, Annals of the Missouri Botanical Garden, 2005 92(3): 425437. 